\title{
Design of High Altitude Propeller Using Multilevel Optimization
}

\author{
Xinqiang Liu*,‡, Weiliang $\mathrm{He}^{*, \S}$ and Fengmei Wei ${ }^{\dagger, \uparrow}$ \\ ${ }^{*}$ School of Astronautics, Beihang University \\ Beijing 100191, P. R. China \\ ${ }^{\dagger}$ Institute of Mechanics, Chinese Academy of Sciences \\ Beijing 100190, P. R. China \\ $\ddagger_{b u a a \_l x q @ 163 . c o m}$ \\ §heweiliang@buaa.edu.cn \\ 『wfmbuaa@163.com
}

Received 7 April 2018

Accepted 6 December 2018

Published 15 January 2019

\begin{abstract}
An improved D'Angelo optimization framework based on the surrogate model and optimization algorithm is proposed to design and optimize a new high altitude propeller which is applied to the propulsion system of the stratospheric aircraft. The framework adopts a multilevel optimization strategy to determine a high efficiency and light weight propeller. The aerodynamic characteristics of S1223 airfoil are calculated by Computational Fluid Dynamics (CFD) method. The aerodynamic performance of the D'Angelo propeller which is obtained by the first-level optimization is predicted by Blade element momentum (BEM) theory. Then the D'Angelo propeller chord and twist angle, which are regarded as the initial conditions of the second-level optimization, are optimized to achieve the maximum efficiency by Multi-island genetic algorithm (MIGA). In addition, the non-dominated sorting genetic algorithm II (NSGA-II) is applied to maximize the propeller efficiency and minimize the propeller weight. And the Pareto frontier solutions about the efficiency and the blade area are obtained by NSGA-II. What is more, the aerodynamic characteristics of the D'Angelo propeller, the optimization propeller by MIGA, and the optimization propeller by NSGA-II are calculated by CFD simulation and compared with BEM results. It is shown that the CFD results are in fair agreement with BEM results and the aerodynamic performance of the NSGA-II propeller is prior to the MIGA propeller and is close to the D'Angelo propeller. Besides, the NSGA-II propeller is the lightest among them and can satisfy the cruise requirements of the high altitude propeller.
\end{abstract}

Keywords: High altitude propeller; multilevel optimization; blade element momentum theory; MIGA; NSGA-II; CFD simulation.

\section{Introduction}

The stratosphere is defined as the atmospheric region from about $20 \mathrm{~km}$ to $100 \mathrm{~km}$ above the Earth's surface, which is between the highest flight altitude of the plane

$\ddagger$ Corresponding author. 
and the minimum orbit height of the satellite. Many countries compete for the development of the stratospheric aircraft [Colozza (2003); Meng et al. (2017)].

Stratosphere aircrafts are divided into high-speed and low-speed stratosphere aircrafts according to the velocity. Low-speed aircrafts include the stratosphere airship and long-endurance solar unmanned aerial vehicle such as: Pathfinder, Pathfinder Plus, Centurion, HP01, and HP03 [Noll et al. (2004)]. The propulsion units of these low-speed stratosphere aircrafts is the high altitude propeller. The efficiency of the propeller has a great effect on the total efficiency of propulsion system and the propeller needs to have a high propulsive efficiency in the cruise flight Morgado et al. (2015); Liu and He (2017)]. The stratosphere air density is much lower than the ground and the speed of aircrafts is extremely slow, which lead to the low Reynolds number for the propeller and make the propeller efficiency become low [Zheng et al. (2017)]. The air density at $20 \mathrm{~km}$ is one-fourteenth of the sea level and Reynolds number ranges from $10^{4}$ to $10^{6}$. If the section airfoils of the low altitude propellers are used in the high altitude propellers, low Reynolds number may affect the airfoil aerodynamic efficiency. So, it is very necessary to further study the high altitude propeller.

Blade element momentum (BEM) McCrink and Gregorv (2017); Morgado et al. (2014); Jiao et al. (2018)] theory is widely used to predict the aerodynamic performance of the propeller and the prediction accuracy of BEM depends on the reliability of the airfoil aerodynamic characteristics data. So knowing the chord distribution, twist angle distribution, and aerodynamic data of the section airfoil, the thrust, torque, and efficiency of the propeller can be obtained by using BEM theorv. The minimum induced losses theorv was proposed bv Betz [1919] and Goldstein [1929] to design a propeller at the beginning of the 20th century and then was developed by several famous scholars [Adkins and Liebeck (1994); Larrabee (1979)]. So, BEM theory is used to calculate the aerodynamic performance of the propeller.

With the development of the computer technology, numerical simulation is widely used to calculate the performance of the propeller. Rahmati 2009] adopted the modified $k-\varepsilon$ turbulence model to simulate the four-bladed propeller model. The results of the numerical calculations are in reasonable agreement with the experimental data. Sodja et al. 2012] carried out the computational fluid dynamics analysis method to calculate the rotating propeller. The $k-\varepsilon$ turbulence model was adopted. CFD analysis was used as a post-analysis method for aerodynamic characteristics calculation of the high altitude propeller. Mieloszyk et al. 2013] performed the Spalart-Allmaras and $k-\omega$ with the shear-stress transition (SST) turbulence models to simulate the turbulence of the contra-rotating propeller. By comparison with wind tunnel experimental results, S-A and $k-\omega$ SST turbulence models yielded accurate results. Morgado et al. 2014] applied $k-\omega$ shear-stress transport turbulence model to calculate an APC $10 \times 7$ inches propeller. He selected the SIMPLE algorithm and second-order upwind scheme. CFD results show the real trend. Li et al. [2015] employed $k-\omega$ SST turbulent model to simulate the turbulence of a two-blade propeller. The results of CFD calculation are in good agreement 
with experimental results of thrust and torque coefficients. Kwon et al. 2015] used the $k-\omega$ SST turbulence model to predict the aerodynamic characteristics of the commercial propeller. The thrust predicted by CFD analysis was about $6 \%$ lower than experiment, whereas the torque was about $14 \%$ lower than the experimental data. Morgado et al. [2015] applied the $k-\omega$ SST turbulence model to simulate the turbulence and the performance of the high altitude propeller was obtained. Qin et al. [2018] applied the $k-\omega$ SST turbulence model to calculate a worldwide employed ducted propeller and verified the numerical simulation. So, the aerodynamic performance of the optimal propeller can be verified by Computational Fluid Dynamics (CFD) method.

D'Angelo et al. 2002] integrated the vortex theory, wing theory, and momentum theory to calculate the aerodynamic performance of the propeller and proposed the inverse design theory of the propeller. Besides, the maximum efficiency of the propeller could be obtained in the context of knowing the required thrust, the forward velocity, propeller rotation speed, and the flight altitude. Romeo et al. 2012 adopted D'Angelo's method to design a high efficiency two-blade propeller for the ENFICA-FC project [Romeo et al. (2007)] and the propeller was tested and validated by the flight experiment. This two-blade propeller showed a good agreement between the theoretical and experimental data.

D'Angelo inverse design method is mainly applied to design the low altitude propellers. In this paper, the D'Angelo method is firstly adopted to design the high efficiency stratosphere propeller. The chord and twist angle distributions of the high altitude propeller are obtained. Sometimes the chord distribution may be relatively large and may not satisfy the requirement of the engineering, making the propeller very heavy. Light weight and high efficiency propeller is the goal for the low-speed stratosphere aircrafts. To overcome the disadvantage of the D'Angelo method and obtain the high efficiency and lightweight propeller, an improved D'Angelo optimization framework based on surrogate model and optimization algorithm is proposed in this paper. The surrogate model is established by Design of Experiments (DOE) method [Yin et al. (2011); Berci et al. (2014); Zhang et al. (2017)]. Latin hypercube sampling (LHS) method Mackav et al. (1979)] is used to train the surrogate model. The optimization algorithms are Multi-island genetic algorithm (MIGA) Zhao et al. (2015)] and non-dominated sorting genetic algorithm II (NSGA-II) Bekele and Nicklow (2007)]. The thrust, torque and efficiency of D'Angelo propeller obtained by the first-level optimization are calculated by BEM theory. These aerodynamic samples are approximated by the Response Surface Methodology (RSM) model [Jin et al. (2001); Yin et al. (2011); Hou et al. (2014)]. Single objective optimization is used to obtain the maximums efficiency by changing the chord and twist angle distributions of D'Angelo propeller. After optimization, the improvement of the efficiency is not obvious. It is indicated that the efficiency of D'Angelo propeller is relatively decent. Besides, the chord and twist angle optimized by MIGA is more reasonable and the blade area is smaller than D'Angelo propeller. So, the multiobjective optimization design, which integrates the aerodynamic parameters and 
the blade area, is adopted to optimize the high altitude propeller. The Pareto frontier results of efficiency and blade area of the high altitude propeller are obtained by NSGA-II and discussed in detail.

In this paper, CFD method is applied to perform numerical simulations of the propellers obtained by D'Angelo method and multilevel optimization framework. The results are compared with the BEM aerodynamic data to validate the prediction accuracy of BEM theory.

This paper is organized into six main sections. The Blade Element Momentum theory and the improved D'Angelo optimization framework are outlined in Sec. 2 , Next, the aerodynamic characteristics of S1223 airfoil are analyzed and calculated in Sec. 3. In Sec. 4, the first-level optimization is used to design the D'Angelo propeller. The aerodynamic characteristics database of S1223 airfoil are established, which are taken into the BEM theory, and CFD simulation is adopted to verify the aerodynamic performance of the D'Angelo propeller. Besides, the second-level optimization based on the surrogate model and optimization algorithm is applied to optimize the D'Angelo propeller. And MIGA is introduced to solve the single objective optimization problem of the high altitude propeller and NSGA-II is applied to solve the multi-objective optimization problem. Finally, the optimization results of the high altitude propeller using MIGA and NSGA-II are discussed and CFD calculation is used to validate the optimization propellers in Sec 5

\section{Propeller Blade Theory}

\subsection{Blade element momentum model}

In the blade-element method, a propeller blade is divided into a number of sections along its span. Each element is treated as a two-dimensional airfoil. The basic forces acting on the airfoil are shown in Fig. 1 [Dorfling and Rokhsaz (2014)].

$v_{\infty}$ is forward speed of the propeller. $N_{B}$ is blade number of the propeller. $n$ is angular velocity of the propeller. $V$ is total kinematic velocity of the actual airflow. $b$ is chord of the propeller, $v_{a}$ is axial induction velocity and $v_{t}$ is tangential induction velocity. $\beta$ is pitch angle. $\alpha_{i}$ is defined as the interference angle. $\varphi$ is actual airflow. $r$ is radial coordinate. $a$ is axial induction factor and $a^{\prime}$ is tangential induction factor.

The thrust $\mathrm{d} T$ and torque $\mathrm{d} M$ contribution of each element can be expressed in terms of the elemental lift $\mathrm{d} L$ and drag $\mathrm{d} D$.

$$
\begin{aligned}
d L & =\frac{1}{2} \rho V^{2} C_{L} b d r \\
d D & =\frac{1}{2} \rho V^{2} C_{D} b d r \\
d T & =d L \cos \left(\varphi_{0}+\alpha_{i}\right)-d D \sin \left(\varphi_{0}+\alpha_{i}\right) \\
& =\frac{1}{2} \rho v_{\infty}^{2} \frac{N_{B} b(1+a)^{2}}{\sin ^{2} \varphi} C_{a} d r
\end{aligned}
$$




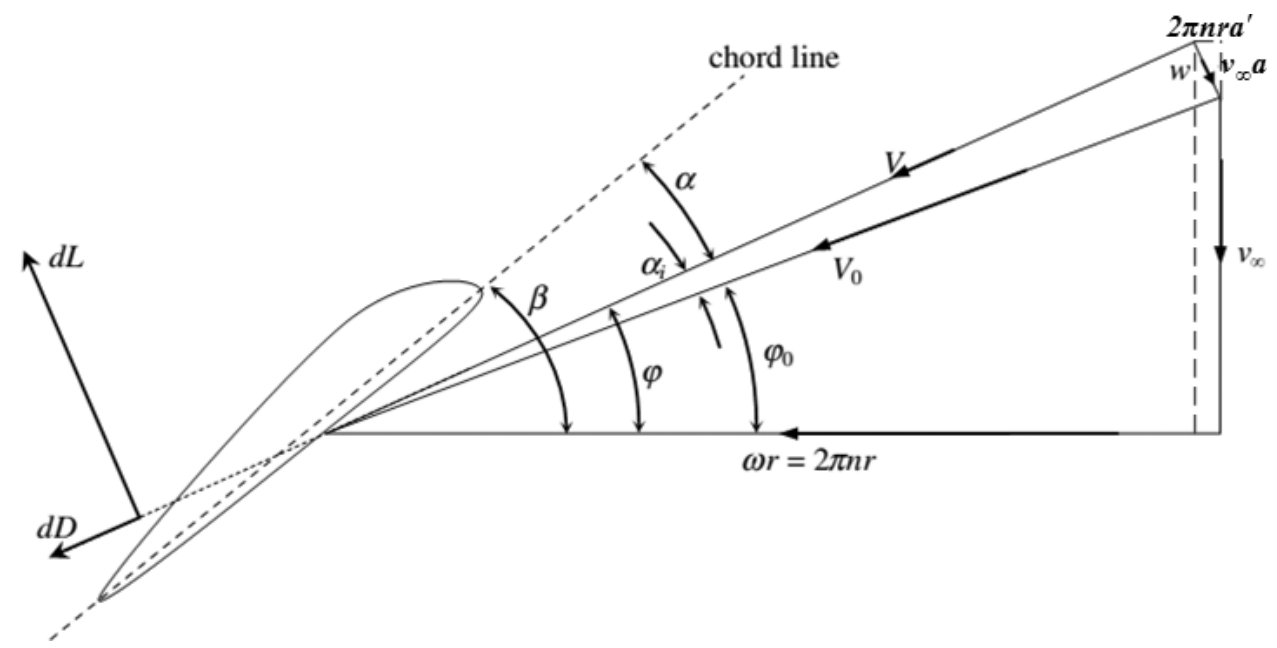

Fig. 1. Schematic of the local flow.

$$
\begin{aligned}
d M & =r d F=r\left(d L \cos \left(\varphi_{0}+\alpha_{i}\right)+d D \sin \left(\varphi_{0}+\alpha_{i}\right)\right) \\
& =\frac{1}{2} \rho v_{\infty}^{2} \frac{N_{B} b(1+a)^{2}}{\sin ^{2} \varphi} r C_{t} d r
\end{aligned}
$$

where $C_{a}$ and $C_{t}$ are related to the local lift coefficient $C_{L}$ and drag coefficient $C_{D}$ of the airfoil section by the following matrix:

$$
\left(\begin{array}{l}
C_{a} \\
C_{t}
\end{array}\right)=\left(\begin{array}{cc}
\cos \varphi & -\sin \varphi \\
\sin \varphi & \cos \varphi
\end{array}\right)\left(\begin{array}{l}
C_{L} \\
C_{D}
\end{array}\right) .
$$

The momentum theory equations are given as

$$
\begin{aligned}
d T & =4 \pi r \rho v_{\infty}^{2} a(1+a) d r, \\
d M & =4 \pi r^{2} \rho v_{\infty}(2 \pi r n) a^{\prime}(1+a) d r .
\end{aligned}
$$

Equating Eqs. (6) and (7) with Eqs. (3) and (4), the induced velocity components can be obtained.

$$
\begin{aligned}
& a=\frac{v_{a}}{v_{\infty}}=\left(\frac{4 F \sin ^{2} \varphi}{\sigma C_{a}}-1\right)^{-1}, \\
& a^{\prime}=\frac{v_{t}}{2 \pi n r}=\left(\frac{4 F \sin \varphi \cos \varphi}{\sigma C_{t}}+1\right)^{-1},
\end{aligned}
$$

where $\sigma$ is the local solidity of the propeller and it is defined by Eq. (10).

$$
\sigma=\frac{N_{B} b}{2 \pi r} .
$$

$F$ is the momentum loss factor and it is described by Prandlt Glauert (1963)]. Setting the arbitrary $a$ and $a^{\prime}$ for the first iteration, $\varphi$ can be computed. The lift 
and drag coefficients of the airfoil section at the angle of attack can be obtained. The induction factors can be updated by Eqs. (8) and (9). And they are compared with the previous iteration. If the difference is less than the set, the iteration stops. The suitable induction factors are obtained.

It is assumed that the number of propeller blades is infinite in the vortex theory. But in fact the number of blades is finite. So the momentum functions need to be modified. Prandlt proposed momentum loss factor $F$ [Glauert (1963)].

$$
F=\frac{2}{\pi} \arccos \left(e^{-f}\right)
$$

where $f$ is

$$
f=\frac{N_{B}}{2} \frac{R-r}{R \sin \phi_{t}},
$$

where $\phi_{t}$ is the flow angle of the blade tip.

Dimensionless thrust and power coefficients are calculated from the following equations:

$$
\begin{aligned}
& C_{T}=\frac{T}{\rho n^{2} D^{4}}, \\
& C_{P}=\frac{2 \pi n M}{\rho n^{3} D^{5}} .
\end{aligned}
$$

The advance ratio is presented in (15). The propeller efficiency is computed through (16)

$$
\begin{aligned}
& J=\frac{v_{\infty}}{n D}, \\
& \eta=\frac{C_{T}}{C_{P}} J .
\end{aligned}
$$

\subsection{Improved D'Angelo optimization framework for high altitude propeller}

D'Angelo et al. 2002] combined the Vortex theory with the Wing theory to calculate the aerodynamic parameters of a propeller. The maximum efficiency propeller under condition of the required thrust $T$, the forward speed, propeller angular velocity and flight altitude could be designed and the maximum efficiency propeller could be obtained when all the airfoils along the blade span were at their maximum efficiency angle of attack. The chord and twist angle distributions of the propeller can be calculated by D'Angelo method. Actually the chord distribution is relatively large and the blade area is large. In general, the larger the blade area is, the heavier the weight of the propeller is. So, a multilevel optimization strategy is put forward in the paper to design the high altitude propeller. D'Angelo method is regarded as the first-level optimization in order to obtain the initial high altitude propeller which can satisfy the cruise requirements. The goal of the second-level optimization 

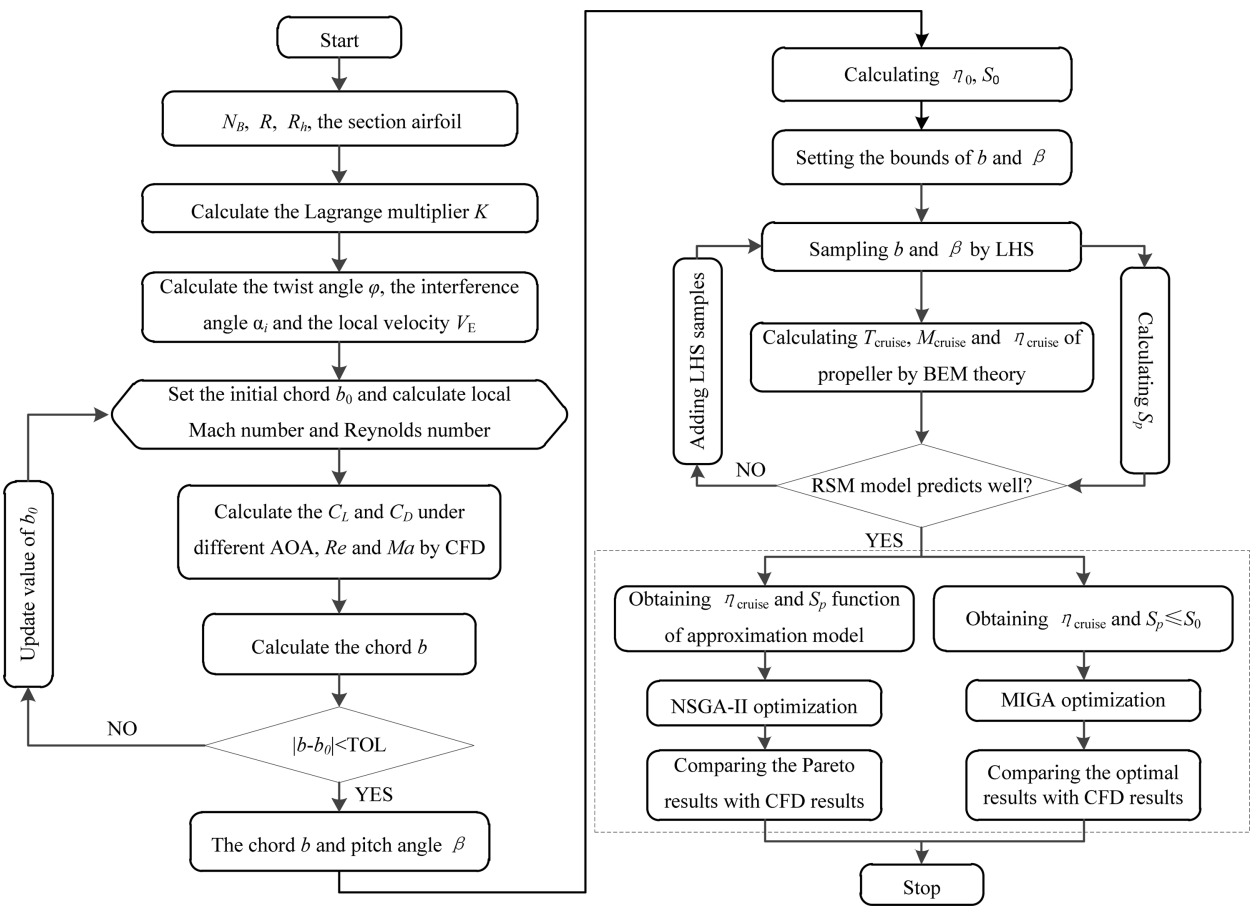

Fig. 2. Improved D'Angelo optimization framework for the high altitude propeller.

based on surrogate model and optimization algorithm is to get the high efficient and lightweight propeller. The improved D'Angelo optimization framework is shown in Fig. 2. The detailed optimization steps are presented as follows:

(1) blade number $N_{B}$, the radius of propeller $R$, the hub radius $R_{h}$, the airfoil distribution number along the blade and the section airfoil.

(2) the Lagrange multiplier $K$ is determined by Eq. (17). This equation can be solved numerically by a bisection method.

$$
\begin{aligned}
\frac{T}{4 \pi \rho v_{\infty}^{2}} & =\int_{R_{h}}^{R}\left(K_{1}+K_{1}^{2}\right) F r d r \\
K_{1} & =\frac{K}{1+\left(\frac{v_{\infty}}{\omega r}\right)^{2}(1+K)^{2}},
\end{aligned}
$$

where $F$ is Prandlt momentum loss factor.

(3) Confirming the distribution of twist angle and the local velocity of the section airfoil. The twist angle $\varphi$ and the interference angle $\alpha_{i}$ are given by

$$
\varphi=\arctan \left(\frac{\lambda}{x_{i}}(1+K)\right),
$$


X. Liu, W. He \& F. Wei

$$
\begin{aligned}
\alpha_{i} & =\arctan \left(\frac{K \sin \varphi \cos \varphi}{1+K \cos ^{2} \varphi}\right), \\
\hat{V}_{E} & =\sqrt{1+\left(\frac{x_{i}}{\lambda}\right)^{2}} \cos \alpha_{i},
\end{aligned}
$$

where $x_{i}$ is the nondimensional radius, $\lambda=\frac{v_{\infty}}{\omega R}$ is speed ratio.

(4) Setting the initial chord $b_{0}$ of the section airfoil. The local Mach number and Reynolds can be calculated.

$$
\begin{aligned}
\mathrm{Ma}_{i} & =\hat{V}_{E} \mathrm{Ma}, \\
\operatorname{Re}_{i} & =\hat{V}_{E} b_{0} \mathrm{Re}, \\
\operatorname{Re} & =\frac{\rho v_{\infty}}{\mu}, \\
\mathrm{Ma} & =\frac{v_{\infty}}{c},
\end{aligned}
$$

where Ma is the Mach number of the undisturbed flow upstream propeller, Re is the Reynolds number whose reference length is $1 \mathrm{~m}$.

(5) Establishing the aerodynamic database of the section airfoil and calculating the lift coefficients and drag coefficients under different AOAs, Re and Ma. And maximum lift-drag ratio of the section airfoil can be obtained. The chord and pitch angle are given by

$$
\begin{aligned}
& b=\frac{8 \pi R}{N_{B}} \frac{x_{i} k_{P} \tan \alpha_{i} \sin \varphi}{C_{L}\left(\alpha_{\max }\right)-C_{D}\left(\alpha_{\max }\right) \tan \varphi}, \\
& \beta=\varphi+\alpha_{\max } .
\end{aligned}
$$

If $\left(b-b_{0}\right)$ can reach the convergence criteria TOL $(0.001 \mathrm{~m})$, we can continue the next step. Otherwise go to step 4.

(6) Repeat steps 4 and 5, we can get the chord and pitch angle of every section airfoil.

(7) Calculating the efficiency and area of the D'Angelo propeller. These data are the initial values of the second-level optimization.

(8) LHS method is applied to train the surrogate model, including the thrust, torque, efficiency, and area of the D'Angelo propeller. RSM models are established.

(9) The MIGA is used to optimize the D'Angelo propeller to obtain the higher efficiency propeller and the NSGA-II is used to optimize the D'Angelo propeller to obtain the light weight and high efficiency propeller.

(10) CFD simulation method is applied to validate the optimization results of improved D'Angelo optimization framework.

The requirements of the stratospheric aircraft propulsion system are: the advance velocity of the propeller is $30 \mathrm{~m} / \mathrm{s}$, the efficiency is larger than 0.7 and the thrust is larger than $100 \mathrm{~N}$. According to the requirements, the design parameters 
of the high altitude propeller are $D=2.5 \mathrm{~m}, N_{B}=3, n=960 \mathrm{rpm}, v_{\infty}=30 \mathrm{~m} / \mathrm{s}$, $R_{h}=0.1 R$. And the section airfoil is S1223. The detailed propeller design can refer to Liu and $\mathrm{He}$ [2017]. The improved D'Angelo optimization is adopted to design the high altitude propeller.

\section{Aerodynamic Characteristics of S1223}

The air kinematic viscosity of $20 \mathrm{~km}$ is $16.15 \times 10^{-5} \mathrm{~m}^{2} / \mathrm{s}$ and the ground is $1.46 \times 10^{-5} \mathrm{~m}^{2} / \mathrm{s}$. The Reynolds number of $20 \mathrm{~km}$ which belongs to the scope of low Reynolds number is one eleventh of the ground. The laminar flow separation phenomenon of the airfoil can appear under condition of low Reynolds number. It can bring that the lift coefficient increases slowly and the drag coefficient increases rapidly as the angle of attack increases. S1223 is a high lift-drag ratio airfoil and has a good aerodynamic performance under condition of low Reynolds number $[\mathrm{Ma}$ and Liu (2009); Zhang et al. (2017)]. And Tang et al. [2015] has performed a wind tunnel experiment for the counter-rotating propellers using S1223 airfoil. So, S1223 is chosen in this paper. The high altitude propeller is divided into nine section airfoils and every section airfoil is S1223. There are three methods to obtain the aerodynamic data of the airfoil, which are XFOIL program [Drela (1989)], CFD simulation and wind tunnel tests. We can easily gain the aerodynamic data, as long as the Mach number and Reynolds number are appropriate. But Mach number and Reynolds number change a lot from the hub to tip of the propeller. XFOIL program may be no convergence during its execution. And the drag coefficients calculated by means of XFOIL program is smaller than the experiment results. Furthermore wind tunnel tests can obtain the accurate data, but it is very expensive and time-consuming. Therefore, CFD simulation is chosen to calculate the lift and drag data of airfoil.

According to D'Angelo's numerical procedure, firstly setting the range of the chord $b=0.01-0.21 R$. Re and Ma of the section airfoil are presented in Table 1 The Reynolds numbers of the high altitude propeller are in the range of lower Re. We must ensure $Y$ plus $<1$. The airfoil is defined with 240 points around its contour and the complete structure C-type mesh number is 44,000 . The general view of the

Table 1. Ranges of Re, Ma, and AOA for the section airfoil.

\begin{tabular}{crll}
\hline Section airfoil number & \multicolumn{1}{c}{ Re } & \multicolumn{1}{c}{ Ma } & AOA \\
\hline 1 & $2515-52814$ & 0.1101 & $0-10$ \\
2 & $3098.7-65072$ & 0.1357 & $0-10$ \\
3 & $3909.9-82107$ & 0.1712 & $0-10$ \\
4 & $4835.2-101540$ & 0.2118 & $0-10$ \\
5 & $5820.3-122230$ & 0.2549 & $0-10$ \\
6 & $6839-143620$ & 0.2995 & $0-10$ \\
7 & $7878.2-165440$ & 0.345 & $0-10$ \\
8 & $8930.7-187540$ & 0.3911 & $0-10$ \\
9 & $9992.2-209840$ & 0.4376 & $0-10$ \\
\hline
\end{tabular}




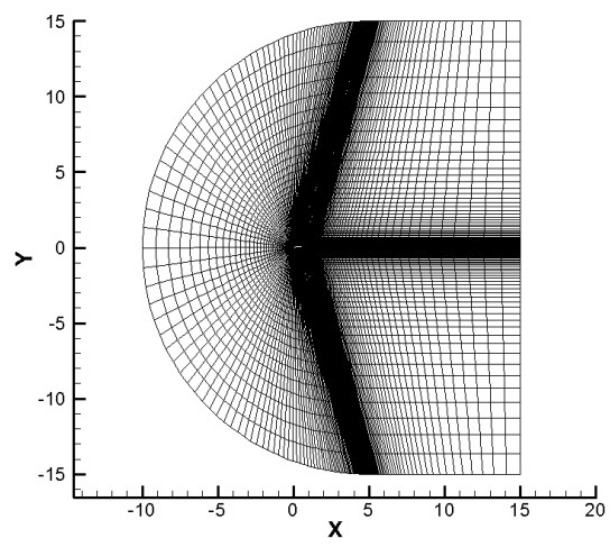

(a)

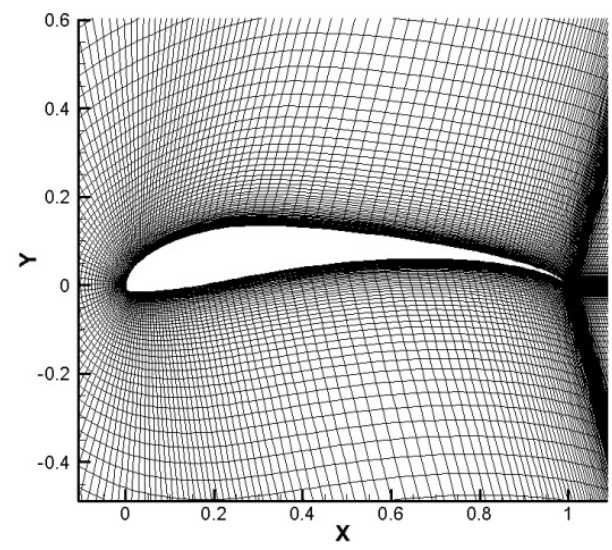

(b)

Fig. 3. (a) The whole computation domain, (b) the mesh around the airfoil.

computational domain and mesh distribution around S1223 airfoil is illustrated in Fig. 3.

The commercial software FLUENT 15.0 is adopted to solve the RANS governing equations. Because of the compressibility of the propeller flow, a density-based solver is used. The $\mathrm{S}-\mathrm{A}$ model is adopted as the turbulence model to calculate the aerodynamic data of S1223. The boundary condition of C-type mesh is defined as the pressure far-field boundary condition. The RANS governing equations are solved implicitly. The flux type is Roe-FDS. A Green-Gauss node-based discretization scheme is adopted in gradient interpolation and the second-order upwind scheme is used for turbulence equations discretization. Convergence is guaranteed by monitoring the relative numerical error of the solution, until it drops below $1.0 \times 10^{-6}$.

The numerical simulations are validated by considering the experiment data of the S1223 airfoil [Selig and Guglielmo (1997)]. The simulation conditions are: Re = $2 \times 10^{5}, \mu=1.7894 \times 10^{-5} \mathrm{~kg} /(\mathrm{m} \cdot \mathrm{s}), \rho=1.225 \mathrm{~kg} / \mathrm{m}^{3}$. The airfoil aerodynamic data predicted at $\mathrm{Re}=2 \times 10^{5}$ using the $\mathrm{S}-\mathrm{A}$ turbulence model and $k-\omega \mathrm{SST}$ turbulence model are compared to the University of Illinois Urbana-Campaign (UIUC) SSelig and Guglielmo (1997)] wind-tunnel measurements in Fig. 4

The results show that the CFD simulations are in good agreement with the experimental data of the S1223 airfoil. From Fig. 4, the lift coefficients calculated by the $k-\omega$ SST model are a little lower than the experimental data and the drag coefficients are much larger than the experiments. And the $\mathrm{S}-\mathrm{A}$ model is more closely approximated to the experimental data than the $k-\omega$ SST model. So, the $\mathrm{S}-\mathrm{A}$ model is accurate and feasible to calculate the aerodynamic coefficients of the S1223 airfoil under the low Mach and low-Reynolds number. Therefore, the S-A model is used to obtain aerodynamic data of every section airfoil.

In order to obtain aerodynamic data of every section airfoil under the different Reynolds numbers and angles of attack, LHS method [Mackay et al. (1979)] is used 

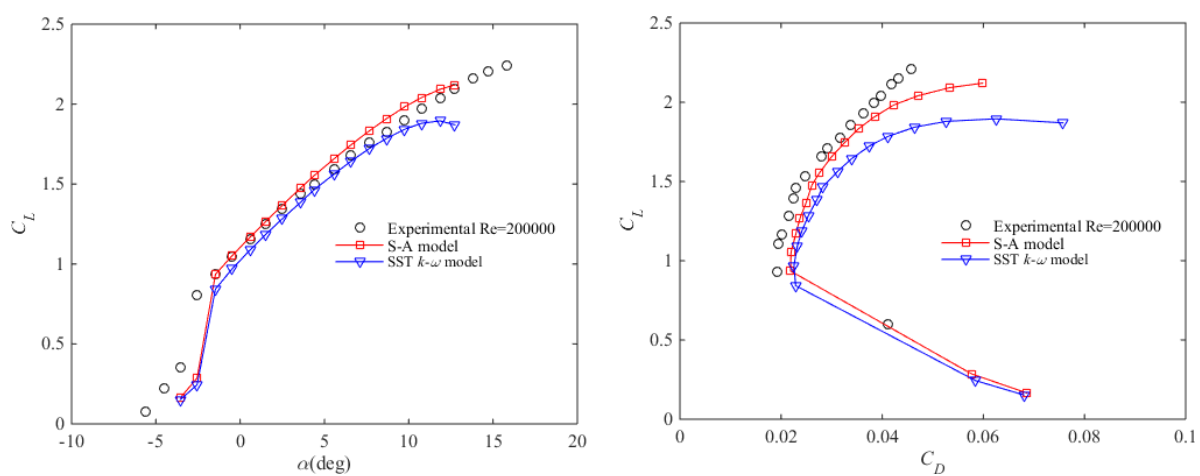

Fig. 4. Lift and lift to drag polar curves of S1223 airfoil at Re $=2 \times 10^{5}$.

to sample the Re and AOA, the number of samples is 300. The aerodynamic data are obtained by the commercial software ISIGHT which can combine MATLAB, MESH software and FLUENT. Sectional lift and drag coefficients for D'Angelo method can be obtained by using MATLAB spline interpolation function. Lift and drag coefficients data of nine sections airfoil are presented in Figs. 5 13.
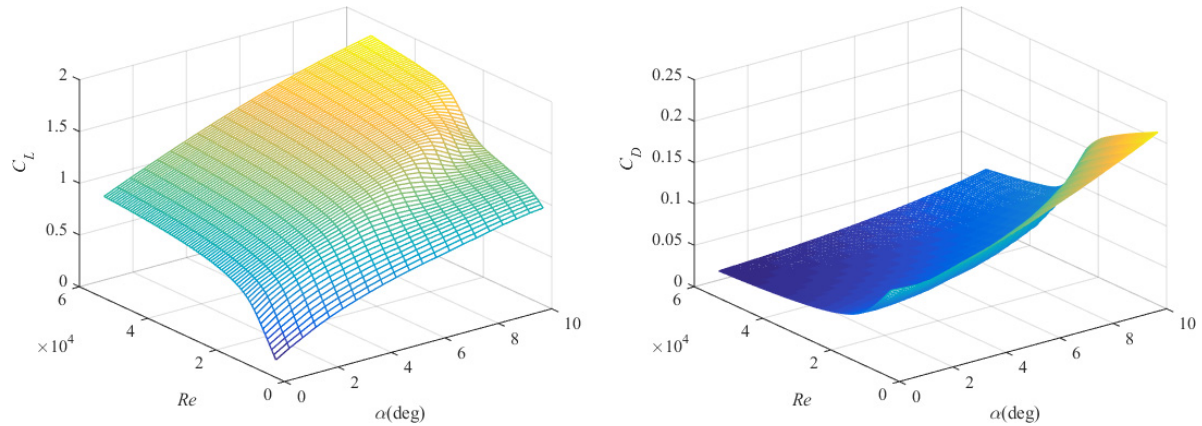

Fig. 5. Lift and drag coefficients versus $\mathrm{AOA}=0-10$ and $\mathrm{Re}=2515-52814$ for $\mathrm{S} 1223$.
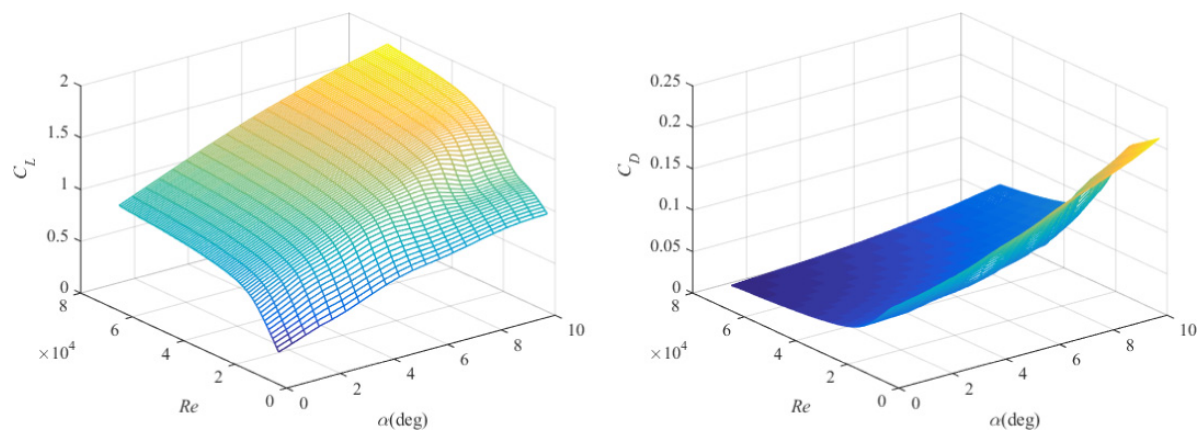

Fig. 6. Lift and drag coefficients versus $\mathrm{AOA}=0-10$ and $\mathrm{Re}=3098.7-65072$ for $\mathrm{S} 1223$. 
X. Liu, W. He \& F. Wei
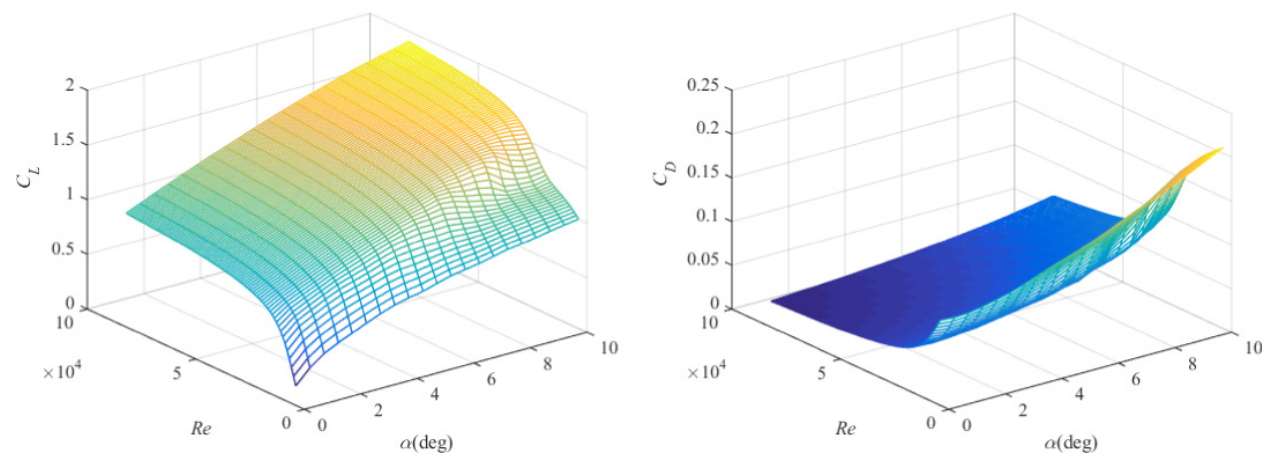

Fig. 7. Lift and drag coefficients versus $\mathrm{AOA}=0-10$ and $\mathrm{Re}=3909.9-82107$ for S1223.
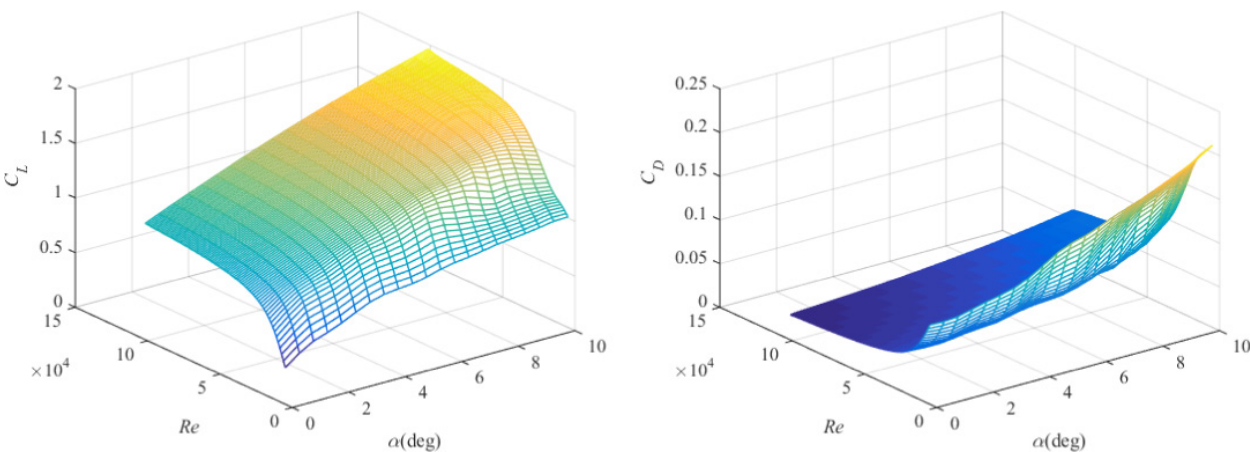

Fig. 8. Lift and drag coefficients versus $\mathrm{AOA}=0-10$ and $\mathrm{Re}=4835.2-101540$ for $\mathrm{S} 1223$.
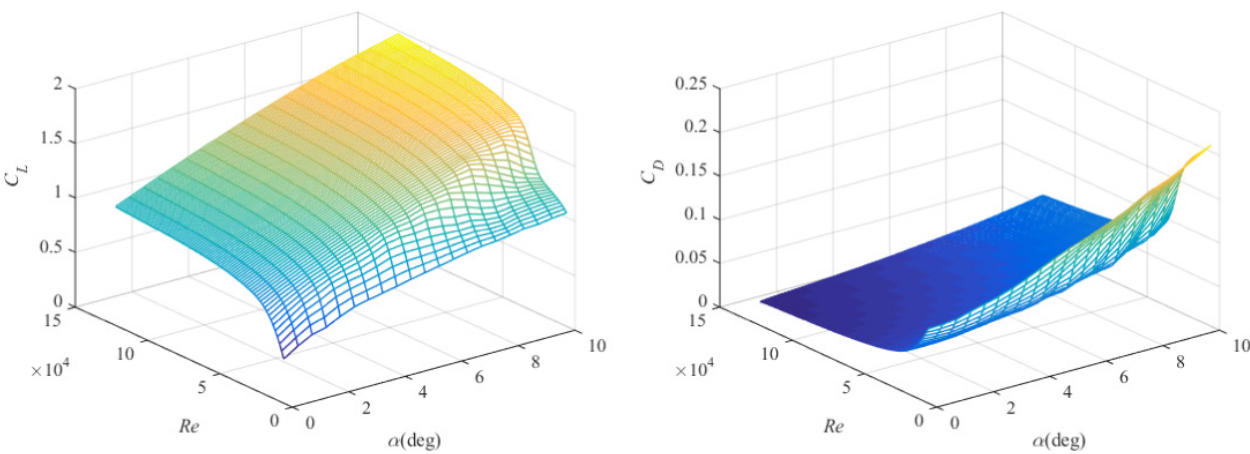

Fig. 9. Lift and drag coefficients versus $\mathrm{AOA}=0-10$ and $\mathrm{Re}=5820.3-122230$ for $\mathrm{S} 1223$. 

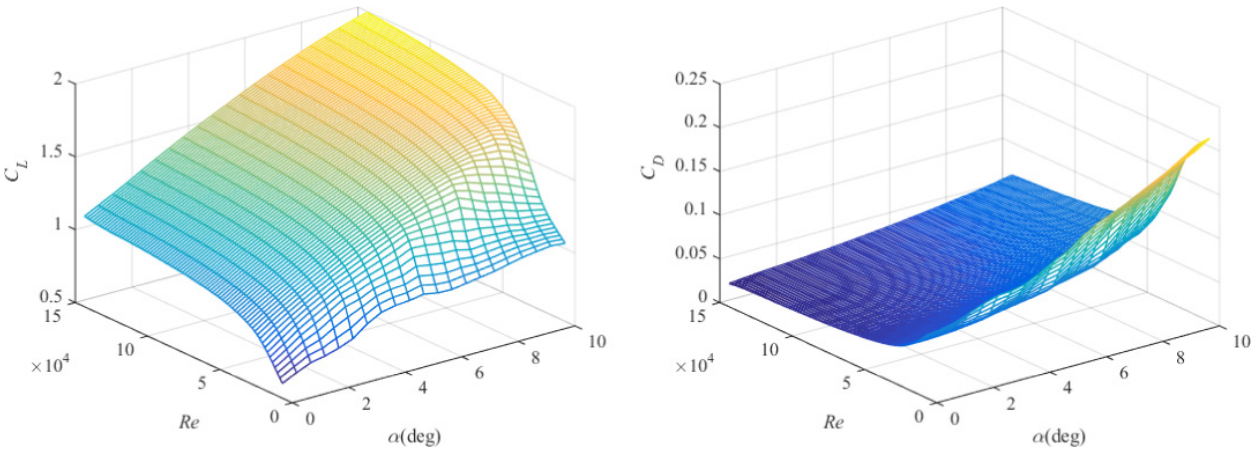

Fig. 10. Lift and drag coefficients versus $\mathrm{AOA}=0-10$ and $\mathrm{Re}=6839-143620$ for $\mathrm{S} 1223$.
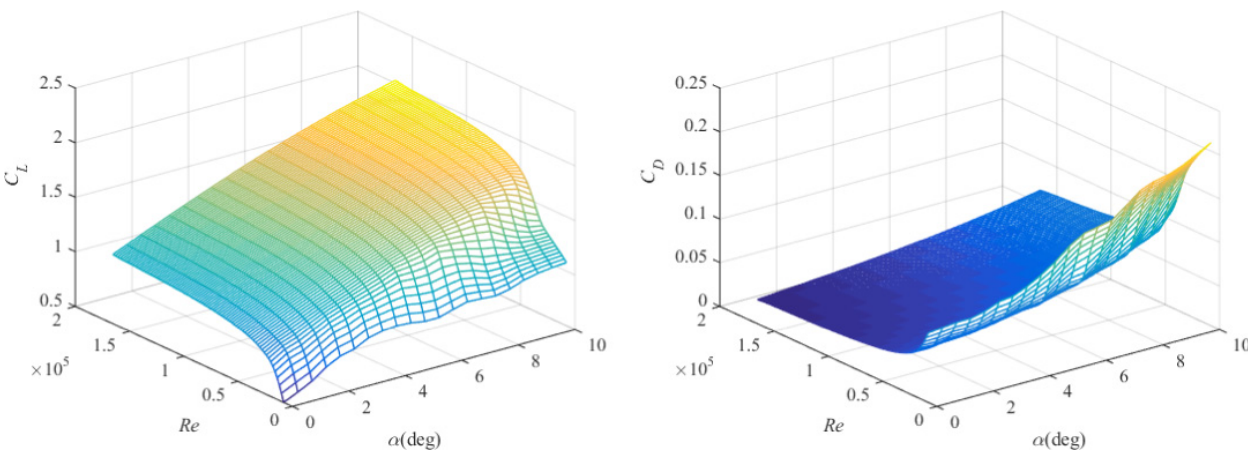

Fig. 11. Lift and drag coefficients versus $\mathrm{AOA}=0-10$ and $\mathrm{Re}=7878.2-165440$ for $\mathrm{S} 1223$.
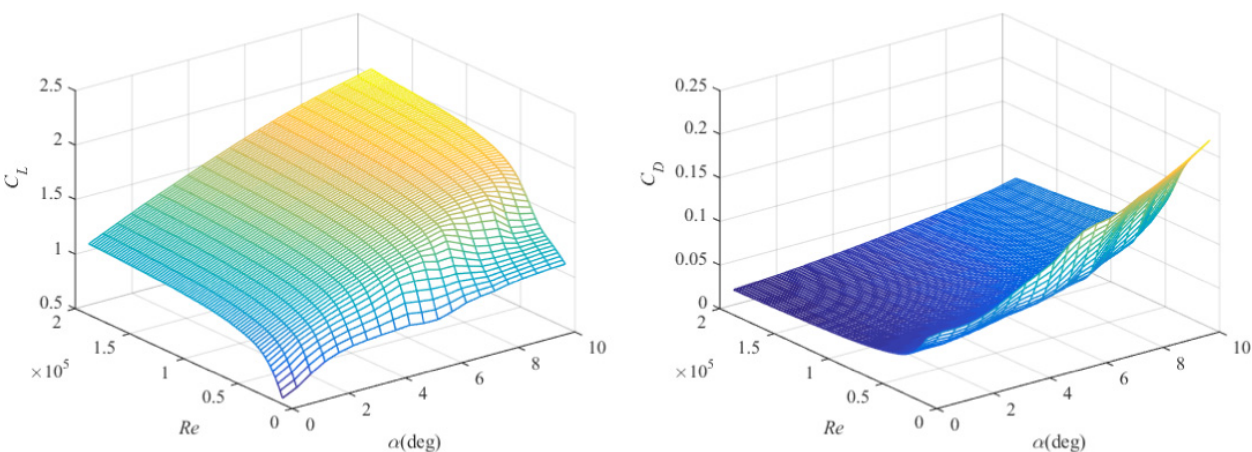

Fig. 12. Lift and drag coefficients versus $\mathrm{AOA}=0-10$ and $\mathrm{Re}=8930.7-187540$ for $\mathrm{S} 1223$. 

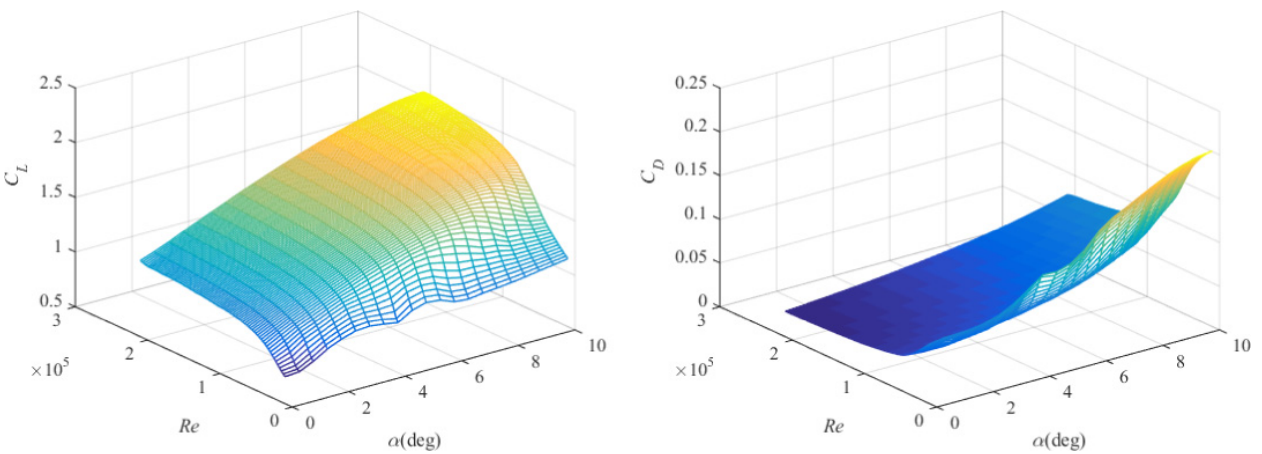

Fig. 13. Lift and drag coefficients versus $\mathrm{AOA}=0-10$ and $\mathrm{Re}=9992.2-209840$ for $\mathrm{S} 1223$.

\section{Optimal Design of High Altitude Propeller}

\subsection{First-level optimization for high altitude propeller}

The design parameters of the high altitude propeller in Liu and $\mathrm{He}$ [2017] are $T=100 \mathrm{~N}, D=2.5 \mathrm{~m}, N_{B}=3, n=960 \mathrm{rpm}, v_{\infty}=30 \mathrm{~m} / \mathrm{s}, R_{h}=0.1 R$. The first-level optimization goal of the improved D'Angelo optimization framework is to obtain the chord and twist angle distributions of the high altitude propeller. Lift and drag coefficients data of nine sections airfoil in Sec. 3 are used for D'Angelo program. The maximum efficiency of the propeller can be obtained if all the airfoils along the propeller span are at their maximum efficiency angle of attack. The chord and twist angle distributions along the radius of the optimal propeller are shown in Fig. 14 Propeller A represents the high altitude propeller in Liu and He 2017] and Propeller B is the first-level optimization propeller.

The speed triangle distribution of Propeller B is more reasonable than Propeller A. The chord distribution of Propeller B is smaller than at the position of $r / R>0.3$ and its weight is lighter than Propeller A. So, the high efficient propeller
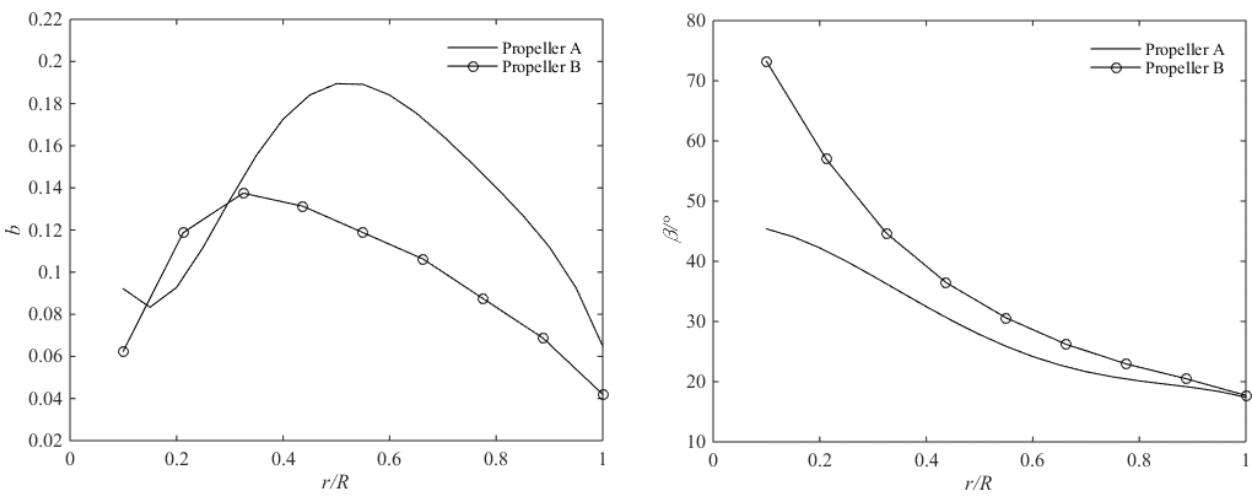

Fig. 14. Chord and twist angle distributions of Propellers A and B. 
Table 2. Calculation results of D'Angelo method.

\begin{tabular}{lccrcc}
\hline & $v_{\infty}(\mathrm{m} / \mathrm{s})$ & $n(\mathrm{rpm})$ & $T(\mathrm{~N})$ & $M /(\mathrm{Nm})$ & $\eta$ \\
\hline Liu and He [2017] & 30 & 960 & 98.19 & 39.75 & $73.87 \%$ \\
D'Angelo & 30 & 960 & 103.45 & 38.81 & $79.54 \%$ \\
\hline
\end{tabular}

can be achieved by adjusting the chord and twist angle distributions. The calculation results of Liu and $\mathrm{He}$ [2017] and D'Angelo method are listed in Table 2 Under the cruise condition of $n=960 \mathrm{rpm} v_{\infty}=30 \mathrm{~m} / \mathrm{s}$, the thrust is $103.45 \mathrm{~N}$ and the efficiency increases by $5.67 \%$ for D'Angelo method. And the first-level optimization propeller can satisfy the design requirements of the high altitude propeller. The solid model of the optimal propeller is established by CATIA. The three-dimensional models of Propellers A and B are shown in Fig. 15]

The aerodynamic characteristics of the section airfoil S1223 are: Mach number is $0.1-0.8$, Reynolds number is $10,000-100,000$ and angle of attack is $-4-12^{\circ}$. LHS method is used to sample the Ma Re and AOA, the number of samples to establish the approximation model is 300 and 25 random samples are used to analyze the errors of the approximation model. The lift and drag coefficients are calculated by FLUENT. Second-order Response Surface Methodology (RSM) is used to fit these aerodynamic coefficients. RSM is given by Jin et al. [2001], Yin et al. [2011] and Hou et al. 2014.

$$
\begin{aligned}
C_{L}= & A_{0}+A_{1} \alpha+A_{2} \mathrm{Ma}+A_{3} \mathrm{Re}+A_{4} \alpha^{2}+A_{5} \mathrm{Ma}^{2}+A_{6} \mathrm{Re}^{2} \\
& +A_{7} \alpha \mathrm{Ma}+A_{8} \alpha \mathrm{Re}+A_{9} \mathrm{MaRe}, \\
C_{D}= & B_{0}+B_{1} \alpha+B_{2} \mathrm{Ma}+B_{3} \mathrm{Re}+B_{4} \alpha^{2}+B_{5} \mathrm{Ma}^{2} \\
& +B_{6} \mathrm{Re}^{2}+B_{7} \alpha \mathrm{Ma}+B_{8} \alpha \mathrm{Re}+B_{9} \mathrm{MaRe} .
\end{aligned}
$$

The precision of RSM model is assessed by correlation coefficient $\left(R^{2}\right)$ and the root mean square error (RMSE) Yin et al. (2011)]. In general, the closer $R^{2}$.Jin et al. (2001); Yin et al. (2011)] is to 1, the more reliable the RSM model is. The closer

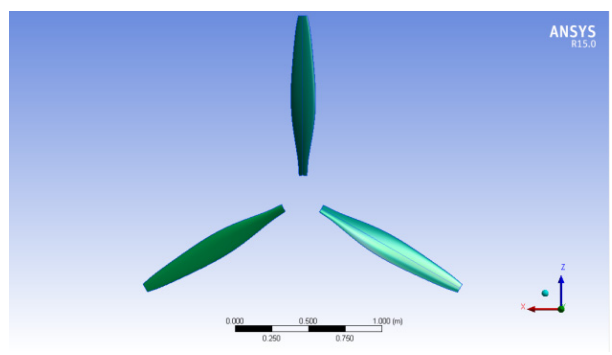

(a)

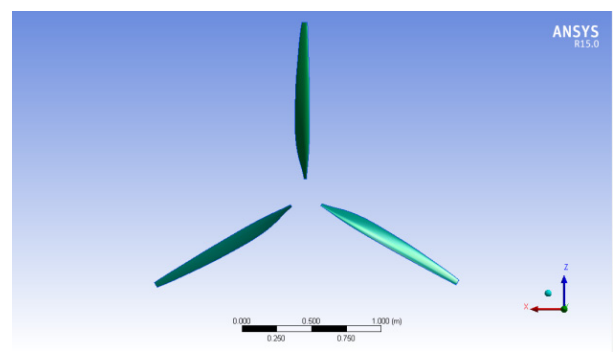

(b)

Fig. 15. (a) Propeller A and (b) Propeller B. 
RMSE is to 0 , the better the fitting precision is. The $R^{2}$ of lift coefficient is 0.95386 and the $R^{2}$ of drag coefficient is 0.97164 . The RMSE of lift coefficient is 0.05666 and the RMSE of drag coefficient is 0.04878 . So, the second-order RSM approximate equations can exactly predict the aerodynamic characteristics of S1223. When $\mathrm{Re}=70,000, \mathrm{Ma}=0.1-0.8$ and $\alpha=-4-12^{\circ}$, the contours of the lift and drag coefficients are shown in Fig. 16. It is clearly seen that the lift coefficient and drag coefficient decline as the Mach number increases. Furthermore, the drag coefficient increases dramatically when the Mach number exceeds 0.55 . When $\mathrm{Ma}=0.3$, $\operatorname{Re}=10,000-100,000$, and $\alpha=-4-12^{\circ}$, the contours of the lift and drag coefficients are presented in Fig. 17 When the angle of attack is small, the Reynolds number has no significant effect on the lift coefficient. And when the angle of attack is big, the Reynolds number has a markedly influence on the lift coefficient. However, the drag coefficient is opposite. When $\alpha=2^{\circ}, \mathrm{Ma}=0.1-0.8$, and $\operatorname{Re}=10,000-100,000$, the contours of the lift and drag coefficients are shown in Fig. 18 It is clearly known
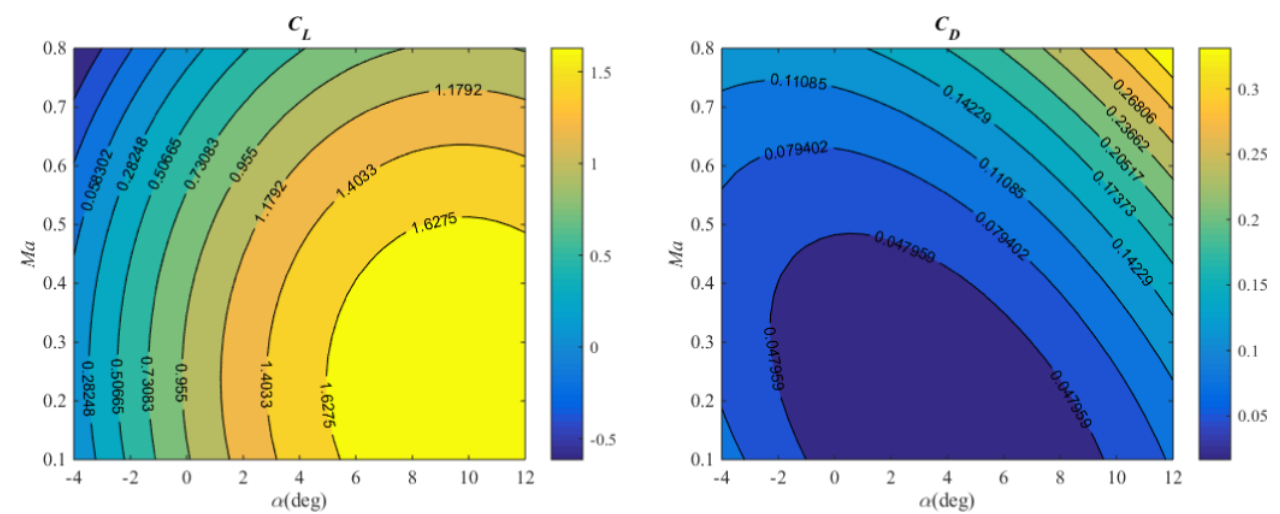

Fig. 16. Lift and drag coefficients of $\mathrm{Re}=70,000$.
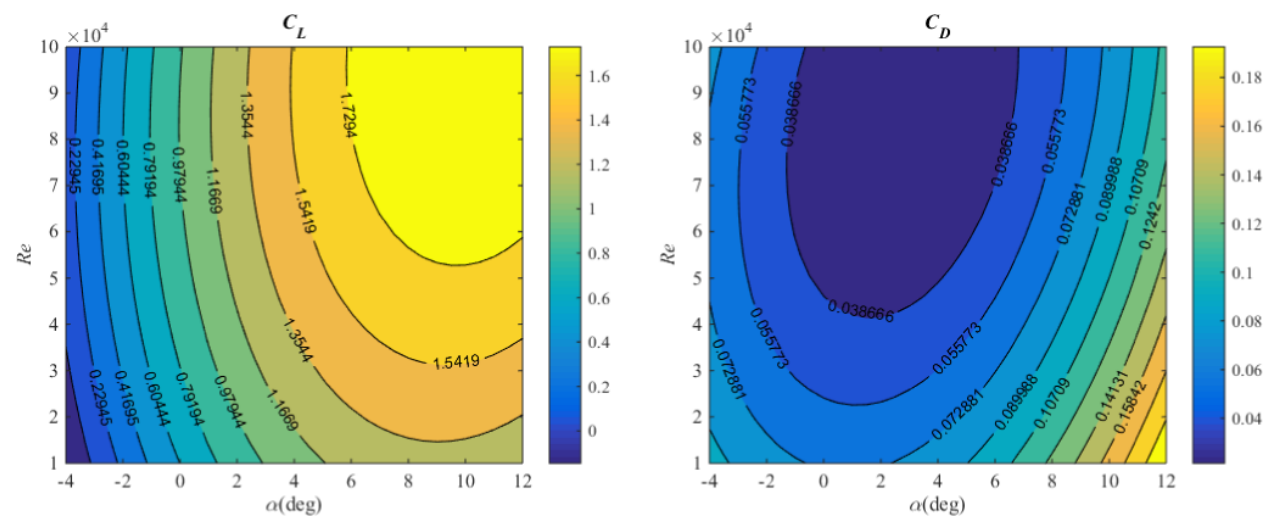

Fig. 17. Lift and drag coefficients of $\mathrm{Ma}=0.3$. 

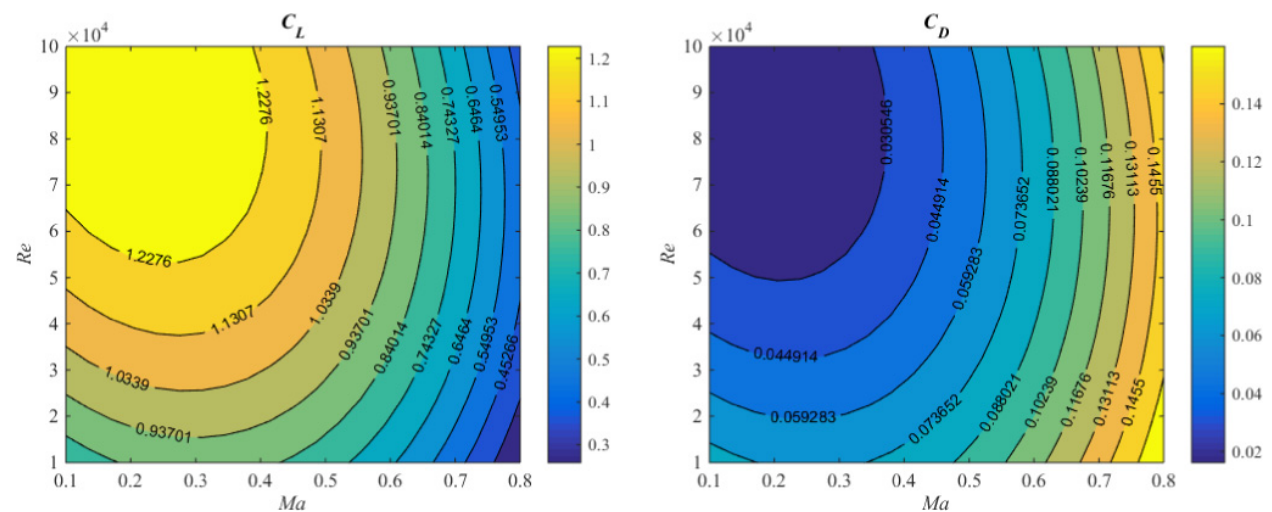

Fig. 18. Lift and drag coefficients of $\alpha=2^{\circ}$.

that the Reynolds number and Mach number have a big effect on the lift and drag coefficients.

These aerodynamic data of S1223 airfoil are applied into the BEM theory. The aerodynamic coefficients of Propeller B can be obtained by BEM theory (Fig. 19). The efficiency of Propeller B is higher than Propeller A.

In order to verify the aerodynamic performance of the first-level optimization propeller, CFD simulations are adopted to study the features of the flow structure and to obtain the aerodynamic coefficients. The computational domain is divided into inner and outer parts. The flow field data between the outer domain and inner domain are exchanged by the Interface. Inner flow field is rotational and the outer is stationary. The upstream of computational domain takes $10 D$. The downstream takes $20 D$. The span-wise length is $10 D$. The boundary conditions of propeller flow field are shown in Fig. 20.

ICEM software is used to draw the mesh of the propeller flow field. In order to ensure $y^{+}<1$, the first layer thickness of inflation is $2 \times 10^{-5} \mathrm{~m}$. The blade is defined by 600 nodes on the spanwise and the element size of the chordwise is $5 \times 10^{-4} \mathrm{~m}$. The whole computational domain is composed of 6.77 million hexagonal and tetrahedral elements clustered around the blade surface as presented in Fig. 21 The number of the outer domain mesh is 1.65 million and the inner domain is 5.12 million. Distribution of the cells on the blade surface is shown in Fig. 22.

The CFD results which are independent on the used mesh are guaranteed by the mesh independency tests. And four different grids are generated to check the results independency at the propeller rotation speed of $960 \mathrm{rpm}$. The number of coarse mesh is 4.21 million, the used mesh is made by 6.77 million and the refined mesh consists of 8.03 million cells. The other mesh is 5.56 million. The CFD results of different grids are listed in Table 3 . The maximum discrepancy between the used mesh and the refined mesh is $0.69 \%$ for the thrust and $1.17 \%$ for the torque of the propeller. Since the accuracy of the used mesh is high and its computation cost is acceptable, the mesh with 6.77 million is adopted in CFD simulation of the propeller. 
X. Liu, W. He \& F. Wei

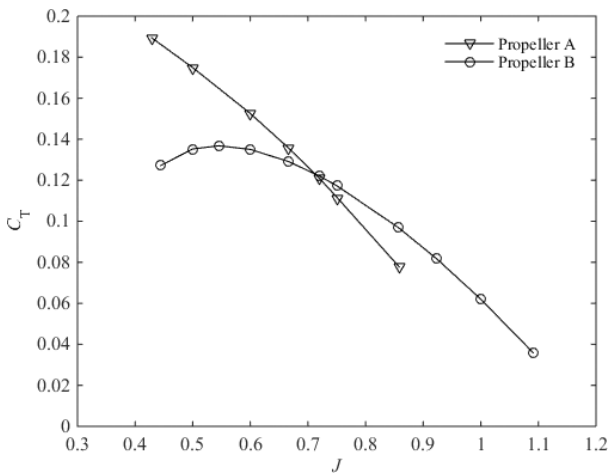

(a)

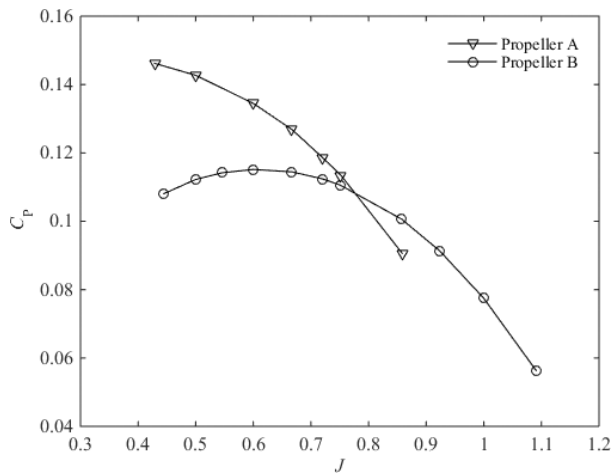

(b)

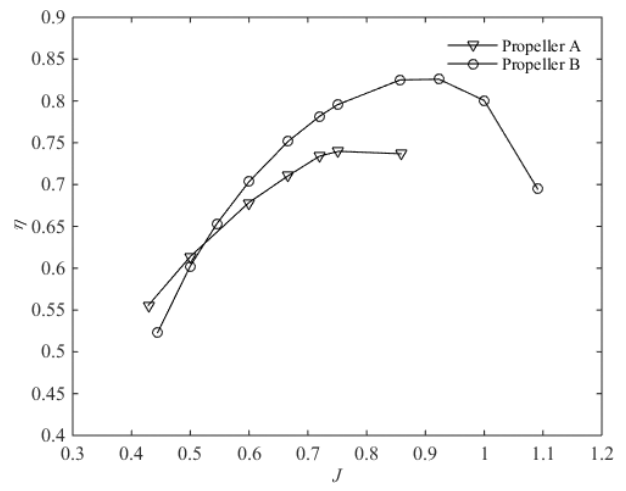

(c)

Fig. 19. Aerodynamic coefficients of Propellers A and B: (a) thrust coefficient, (b) power coefficient, and (c) propeller efficiency.

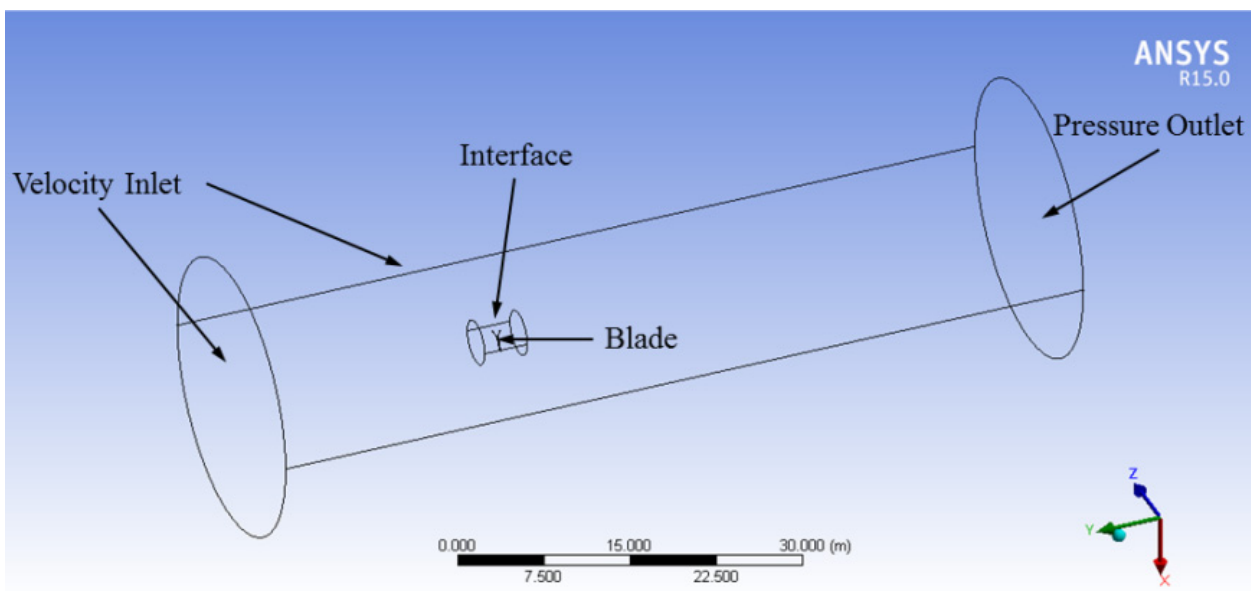

Fig. 20. Computational domain and boundary conditions of the propeller. 


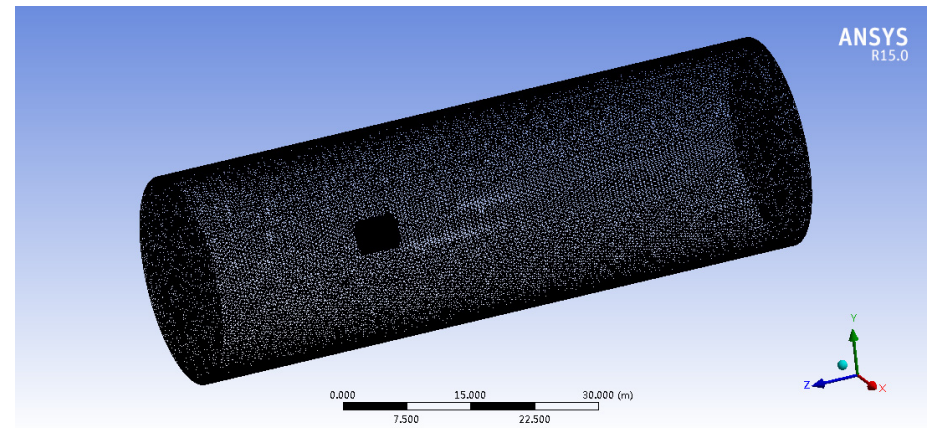

Fig. 21. Meshing of computational domain.

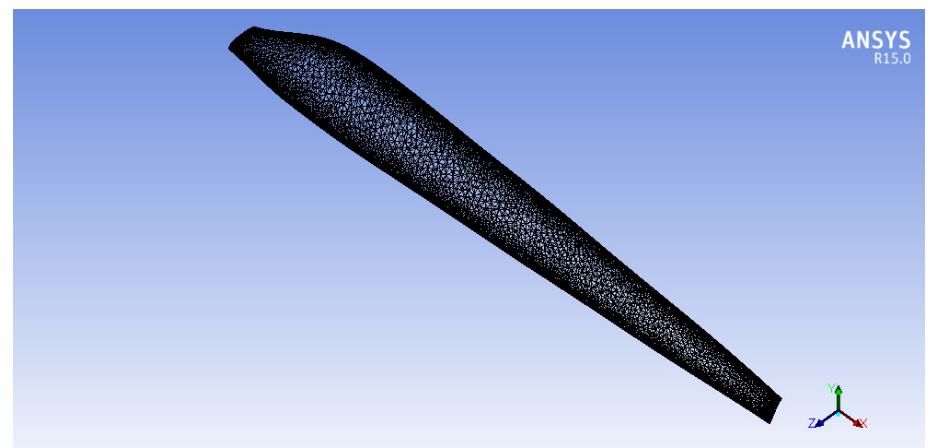

Fig. 22. Distribution of cells on the blade surface.

Table 3. Comparison of the thrust and torque under different grids.

\begin{tabular}{lccccc}
\hline & Cells (million) & $T(\mathrm{~N})$ & $M /(\mathrm{Nm})$ & $\Delta T(\%)$ & $\Delta M(\%)$ \\
\hline Coarse mesh & 4.21 & 95.81 & 38.98 & 1.88 & 3.23 \\
Other mesh & 5.56 & 95.14 & 38.51 & 1.17 & 1.99 \\
Used mesh & 6.77 & 94.69 & 38.2 & 0.69 & 1.17 \\
Refined mesh & 8.03 & 94.04 & 37.76 & 0 & 0 \\
\hline
\end{tabular}

The CFD simulation is completed in FLUENT 15.0. Reynolds-Averaged NavierStokes (RANS) equations are regarded as the governing equations. Rotating coordinate system and pressure-based solver are used. The finite volume method is applied to discretize the governing equations. Spatial discretization is second-order upwind scheme and pressure-velocity coupling is the Coupled algorithm. The courant number is 20. The momentum and pressure explicit relaxation factors are both 0.2. The turbulence model is $k-\omega$ shear-stress transport model which can deal with the time-averaging. The Multiple Reference Frame (MRF) model is used to calculate the rotation motion of the propeller. The atmospheric pressure and temperature in 


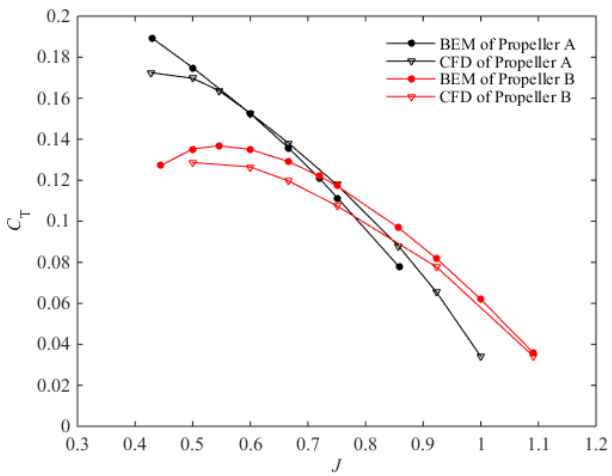

(a)

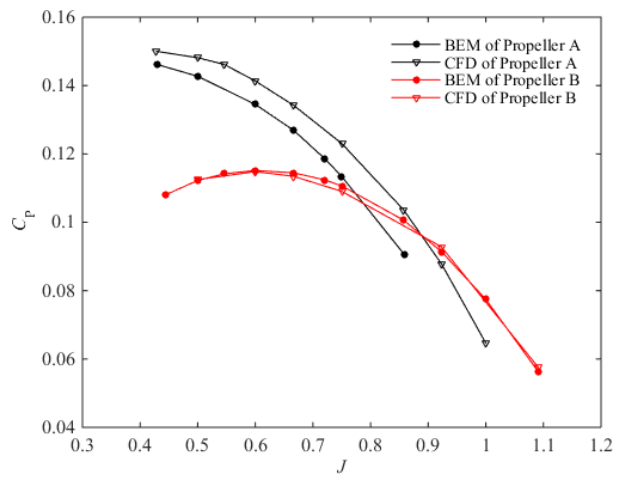

(b)

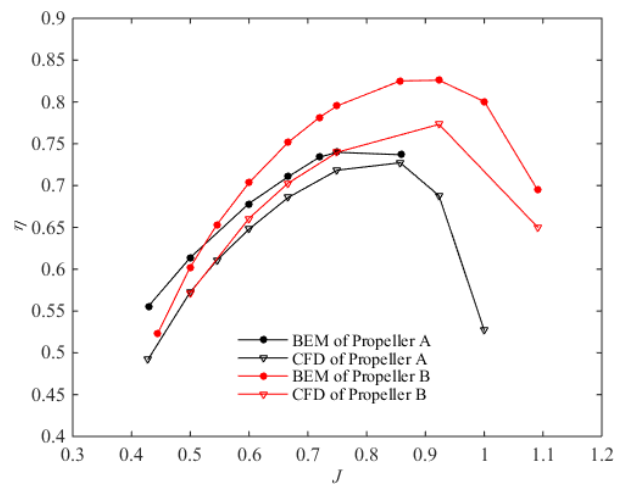

(c)

Fig. 23. Comparison between data calculated by BEM and CFD for Propellers A and B: (a) thrust coefficient, (b) power coefficient, and (c) propeller efficiency.

$20 \mathrm{~km}$ are respectively set as $5474.9 \mathrm{~Pa}$ and $216.7 \mathrm{~K}$. The aerodynamic coefficients of the first-level optimization propeller using the CFD simulation are shown in Fig. 23. The BEM method is widely used due to its effectiveness in design and rapid calculation for the propeller. The BEM theory assumes that there is no momentum exchange existing in the direction of the propeller radius. Moreover, the aerodynamic performance calculated by the BEM theory does not reflect the threedimensional rotating effects of the propeller and the detailed flow characteristics around the propeller are not obtained by the BEM theory. The CFD simulation utilizes the three-dimensional Navier-Stokes equation as the governing equation to obtain the flow characteristics of the propeller. It can obtain the more accurate results than the BEM theory. Sodja et al. 2012] validated that CFD simulation matched the experiment results much more closely than the BEM theory and the BEM results also followed the experiment results very well in terms of trend. Therefore, the CFD simulation is more accurate than the BEM method. However, the CFD simulation consumes much more time and resources to calculate 
the propeller. And the BEM theory can effectively and rapidly design and calculate the aerodynamic characteristics of the propeller. So, these two methods are widely used in the design and optimization of the propeller. Since there is no experimental data, the CFD simulation is used to verify the results of the BEM theory.

Observing Fig. 23, it is possible to conclude that CFD simulation is in fair agreement with BEM. BEM predicts a slightly larger thrust coefficient than CFD at the low advance ratios. The power coefficient of BEM is coincident with CFD. And BEM predicts a slightly larger efficiency than CFD at the high advance ratios. When the advance ratio $J=0.75$, the maximum efficiency difference between BEM calculation and CFD simulation is $5.55 \%$. It is also indicated that BEM can be used to predict the performance of the high altitude propeller and the aerodynamic data of S1223 airfoil calculated by S-A model of FLUENT are correct and reasonable. Besides, the aerodynamic performance of Propeller B using D'Angelo program is better than the original propeller.

\subsection{Second-level optimization for high altitude propeller}

After first-level optimization, the initial high altitude propeller can be obtained. The surrogate models of the thrust, torque, efficiency, and blade area can be established by LHS method. These surrogate models can be used to optimize the propeller. The goal of second-level optimization is to obtain high efficiency and light weight propeller.

\subsubsection{Single-objective optimization of propeller}

Multi-island genetic algorithm (MIGA) is a global optimization algorithm, which uses the principles of natural genetics and natural selection. It utilizes three operators of reproduction, crossover, and mutation. In such method, the population is divided into several sub-populations, whereas traditional genetic algorithm operations are performed on each sub-population separately. Some individuals of the sub-populations are selected and migrated to the different islands, which can maintain the diversity of the population Zhang et al. (2016)].

Since most energy of the stratospheric aircraft is consumed at the cruising flight, the efficiency of the propeller must be improved to reduce energy consumption. The goal in optimizing the chord and the twist of a propeller is to maximize the efficiency. The optimization problem can be expressed as follows:

$$
\begin{array}{ll}
\max & \eta=\frac{T v_{\infty}}{2 \pi n M}, \\
\text { s.t. } & v_{\infty}=30 \mathrm{~m} / \mathrm{s}, \\
& n=960 \mathrm{rpm}, \\
& T \geq 100 \mathrm{~N}, \\
& P_{m} \leq 4,000 \mathrm{~W} .
\end{array}
$$


Under the cruise condition of $n=960 \mathrm{rpm}, v_{\infty}=30 \mathrm{~m} / \mathrm{s}$, the output power of the motor $P_{m} \leq 4,000 \mathrm{~W}$ and the cruise thrust is larger than $100 \mathrm{~N}$, MIGA is used to maximize the cruise efficiency of the propeller. Firstly, the bounds of the chord $b$ and twist angle $\beta$ are ensured. LHS method is used to sample $b$ and $\beta$. Then the BEM theory in Sec. 2 is adopted to calculate the thrust, torque and efficiency of these samples. These aerodynamic samples are approximated by Response Surface Methodology (RSM) model. The approximation model is the objective function of MIGA. Finally the optimal results are verified by CFD simulation.

\subsubsection{Multi-objective optimization of propeller}

The goal of the multi-objective optimization is to minimize or maximize multiple objective functions subject to some constraints. Actually, multi-objective optimization can be translated into the single objective optimization by the scalar method. The multi-objective optimization problem can be described as

$$
\begin{array}{lll}
\min & f_{m}(x), & m=1,2, \ldots, M, \\
\text { s.t. } & g_{j}(x) \leq 0, & j=1,2, \ldots, J, \\
& h_{k}(x)=0, & k=1,2, \ldots, K, \\
& X_{i}^{(L)} \leq X_{i} \leq X_{i}^{(U)}, & j=1,2, \ldots, n,
\end{array}
$$

where $X_{i}$ is the $i$ th design variable, $n$ is the total number of design variables and $X_{i}^{(U)}$ and $X_{i}^{(L)}$ are the bounds. $f_{m}(x)$ is the sub-objective function and $m$ is the number. $g_{j}(x)$ and $h_{k}(x)$ are inequality and equality constraints. $k$ or $j$ is the number of constraints.

The NSGA-II, which is developed based on the original non-dominated sorting genetic algorithm (NSGA), is the multi-objective optimization algorithm which exhibits high performance and has been widely applied in various disciplines. It adopts a quick nondominating sorting approach to discriminate solutions, which is based on the concept of Pareto dominance and optimality Bekele and Nicklow (2007)].

Highly efficient propeller can make full use of the motor output power and the lightweight propeller can reduce energy consumption of the stratospheric aircraft. So, the high efficiency and lightweight propeller needs to be designed. In general, the high altitude propeller is composed of composite materials. The blade weight can be obtained by building the complex structure model. Meng et al. [2017] established the complex structure model and achieved a more lightweight high altitude propeller by GA hierarchical optimization. For simplification of analysis, the blade weight is estimated by the area of the propeller. The area of one blade is as follows:

$$
S_{p}=\int_{R_{h}}^{R} b(r) d r=R \int_{0.1}^{1} b(x) d x .
$$

The chord $b$ and twist angle $\beta$ of the propeller are the design variables. The cruise velocity $v_{\text {cruise }}$ and the rotational speed of propeller $n$ are the equality constraints. 
The cruise thrust $T_{\text {cruise }}$ and the motor output power $P_{m}$ are the inequality constraints. The cruise efficiency $\eta_{\text {cruise }}$ and blade area $S_{p}$ of the propeller are the objective functions. So, the multi-objective optimization of high altitude propeller can be written as

$$
\begin{array}{lll}
\text { Obj } & \max \left(\eta_{\text {cruise }}\right) & \min \left(S_{p}\right), \\
\text { s.t. } & v_{\text {cruise }}=30 \mathrm{~m} / \mathrm{s}, & n=960 \mathrm{rpm}, \\
& T_{\text {cruise }} \geq 100 \mathrm{~N}, & P_{m} \leq 4,000 \mathrm{~W}, \\
& b^{l} \leq b \leq b^{u}, & \\
& \beta^{l} \leq \beta \leq \beta^{u} .
\end{array}
$$

Under the cruise condition of $n=960 \mathrm{rpm}, v_{\infty}=30 \mathrm{~m} / \mathrm{s}, T_{\text {cruise }} \geq 100 \mathrm{~N}$ and $P_{m} \leq 4,000 \mathrm{~W}$, NSGA-II algorithm is used to maximize the cruise efficiency and minimize the blade area of the propeller. Firstly, the bounds of the chord $b$ and twist angle $\beta$ are ensured. Latin hypercube sampling (LHS) method is used to sample $b$ and $\beta$. Then the BEM theory in Sec. 2 is adopted to calculate the thrust, torque and efficiency of these samples. These aerodynamic samples are approximated by RSM model. The cruise efficiency and blade area are the objective functions of NSGA-II algorithm. Finally, the Patero results are compared with the results of CFD simulation.

\subsubsection{Design variables of second-level optimization}

The propeller blade geometry and flight conditions can determine the aerodynamic efficiency of the high altitude propeller. The section airfoil is S1223 and the propeller is divided into nine sections. The total number of design variables is 18 . These 18 design variables can make the optimization complicated and the optimization efficiency is low. So, the amount of design variables must be reduced. In order to make the propeller shape smooth and continuous along the spanwise direction, a fourth-order polynomial can be used to fit the blade chord and twist angle.

$$
\begin{aligned}
& b(x)=b_{0}+b_{1} x+b_{2} x^{2}+b_{3} x^{3}+b_{4} x^{4}, \\
& \beta(x)=\beta_{0}+\beta_{1} x+\beta_{2} x^{2}+\beta_{3} x^{3}+\beta_{4} x^{4} .
\end{aligned}
$$

The fourth-order polynomial can be written as the Bezier curve which can describe the chord and twist angle of the propeller.

$$
\begin{aligned}
& b(x)=(1-x)^{4} b_{0}+4 x(1-x)^{3} b_{1}+6 x^{2}(1-x)^{2} b_{2}+4 x^{3}(1-x) b_{3}+x^{4} b_{4}, \\
& \beta(x)=(1-x)^{4} \beta_{0}+4 x(1-x)^{3} \beta_{1}+6 x^{2}(1-x)^{2} \beta_{2}+4 x^{3}(1-x) \beta_{3}+x^{4} \beta_{4},
\end{aligned}
$$

where $b_{0}, b_{1}, b_{2}, b_{3}$, and $b_{4}$ represent five control points of the chord, $\beta_{0}, \beta_{1}, \beta_{2}, \beta_{3}$, and $\beta_{4}$ represent five control points of the twist angle, the range of $x$ is $[0.1,1]$.

The chord and twist angle of Propeller B in Sec. 3 are regarded as the initial conditions. The bounds of $b_{i}$ and $\beta_{i}$ are listed in Table 4 The upper and lower bounds of the chord and twist angle are shown in Fig. 24. 
Table 4. Ranges of design variables.

\begin{tabular}{cc}
\hline Design variable & Range \\
\hline$b_{0}$ & $-0.0449--0.0299$ \\
$b_{1}$ & $0.2024-0.3149$ \\
$b_{2}$ & $0.0591-0.1299$ \\
$b_{3}$ & $0.1018-0.2444$ \\
$b_{4}$ & $0.0347-0.0595$ \\
$\beta_{0}$ & $77.2-108.99$ \\
$\beta_{1}$ & $31.54-44.52$ \\
$\beta_{2}$ & $23.43-33.07$ \\
$\beta_{3}$ & $19.84-28.02$ \\
$\beta_{4}$ & $14.79-20.88$ \\
\hline
\end{tabular}
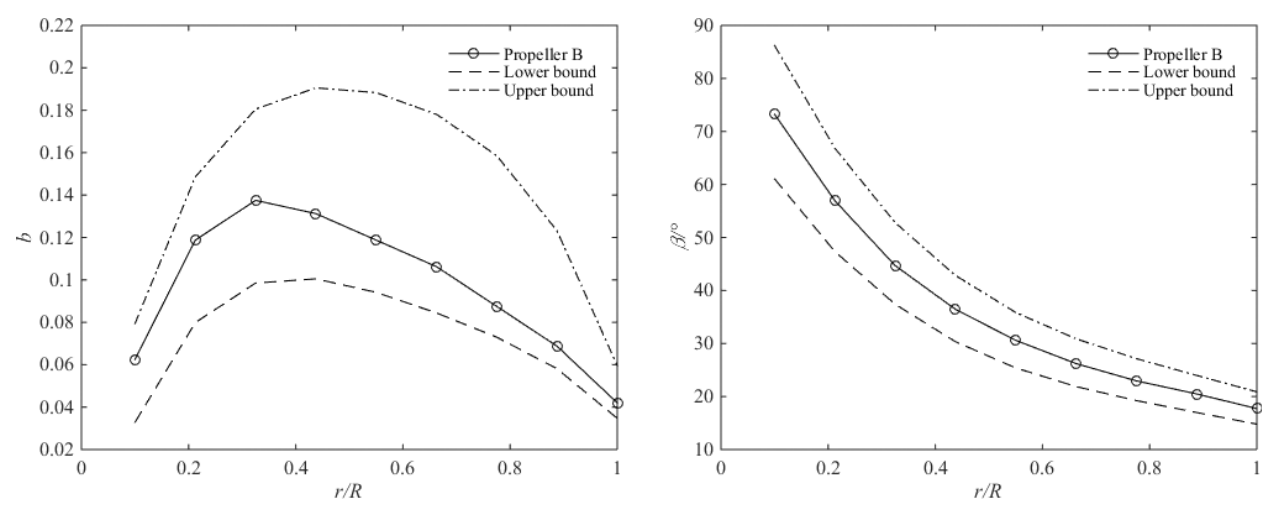

Fig. 24. The upper and lower bounds of the chord and twist angle.

\section{High Altitude Propeller Optimization Results}

Multi-island genetic algorithm is adopted to obtain the optimal high altitude propeller. The parameters of MIGA are presented in Table 5. The first-level optimization propeller using MIGA is regarded as Propeller C. The optimal chord and twist angle are presented in Fig. 25.

Table 5. Parameters of MIGA.

\begin{tabular}{ll}
\hline Parameters & Value \\
\hline Number of generations & 20 \\
Number of islands & 20 \\
Number of individuals on each island & 50 \\
Interval generations of migration & 5 \\
Crossover rate & 0.9 \\
Mutation rate & 0.01 \\
Migration rate & 0.01 \\
\hline
\end{tabular}



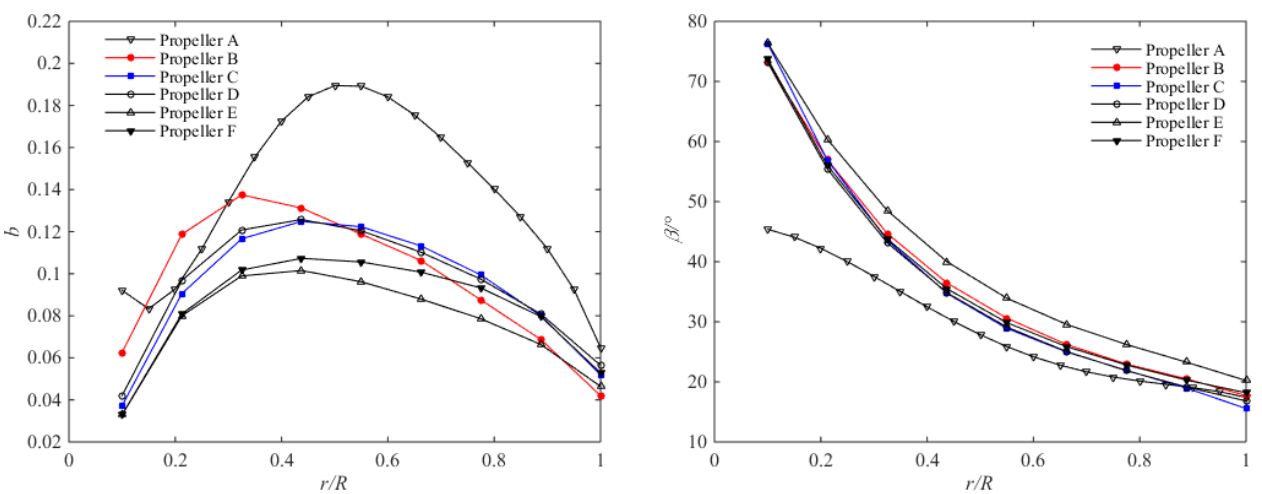

Fig. 25. Chords and twist angles of Propellers A, B, C, D, E and F.

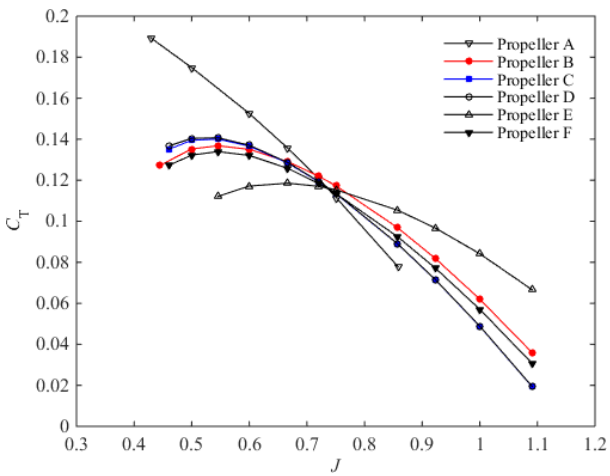

(a)

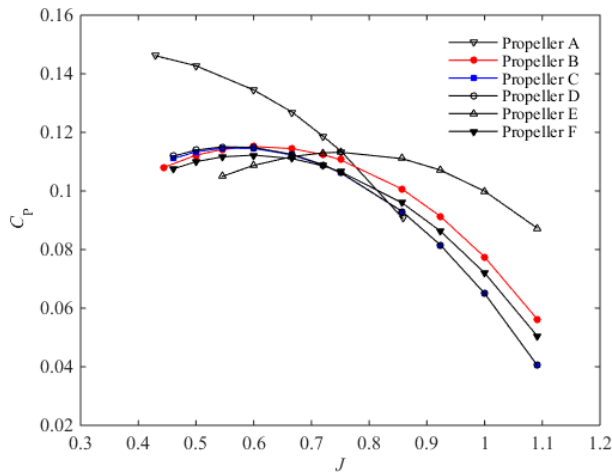

(b)

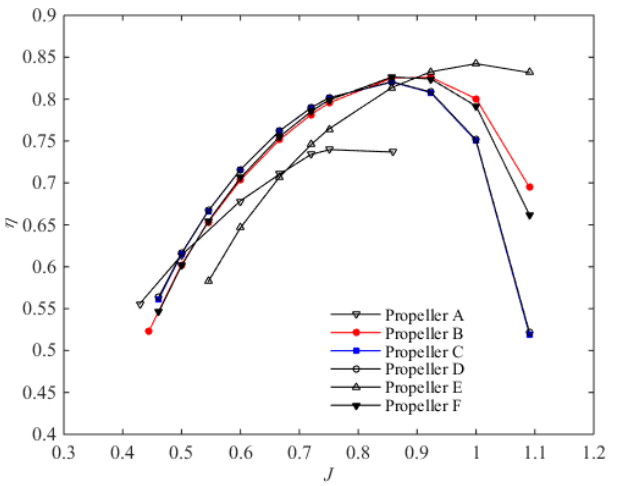

(c)

Fig. 26. Aerodynamic coefficients of Propellers A, B, C, D, E and F: (a) thrust coefficient, (b) power coefficient, (c) propeller efficiency. 
The chord of Propeller C is smaller than Propeller B at $r / R<0.5$. The blade domain from the middle to the tip becomes wide and the twist angle of Propeller $\mathrm{C}$ gets smaller than Propeller B from Fig. 25. The aerodynamic parameters of Propeller $\mathrm{C}$ are calculated by BEM theory and the results are shown in Fig. 26.

When the rotational speed of the propeller is slow, Propeller B can provide the larger thrust and absorb more energy than Propeller C. Similarly, when the rotational speed is fast, Propeller $\mathrm{C}$ can provide the larger thrust and absorb more energy than Propeller B. When $J<0.8$, the efficiency of Propeller C is higher than Propeller B. And the efficiency of Propeller C is $80.15 \%$ and the thrust is $99.93 \mathrm{~N}$ at the cruise flight of $n=960 \mathrm{rpm}, v_{\infty}=30 \mathrm{~m} / \mathrm{s}$.

In Sec. 4, the efficiency and blade area of the high altitude propeller are optimized by NSGA-II. The parameters of NSGA-II are as follows: the population size is 80 and the number of generations is 600 . The optimization results of the propeller are shown in Fig. 27. The monotonicity of the Pareto frontier indicates that the increase of the blade area is accompanied by the increase of the propeller efficiency and there is a conflict in these two objective functions. Three propellers (D, E and F) marked in Fig. 27] are selected to analyze the efficiency and blade area. The chord and twist angle of Propellers D, E and F are shown in Fig. 27] The aerodynamic performances of these propellers are presented in Fig. 26.

As can be seen from Fig. 27. Propeller D is the most efficient propeller among them but the blade area is the largest. Propeller E has the smallest area and efficiency. Propeller F combines the advantages of Propellers D and E and it has the reasonable efficiency and blade area. The optimization results of the propellers $(\mathrm{C}$, $\mathrm{D}, \mathrm{E}$ and F) are listed in Table6.

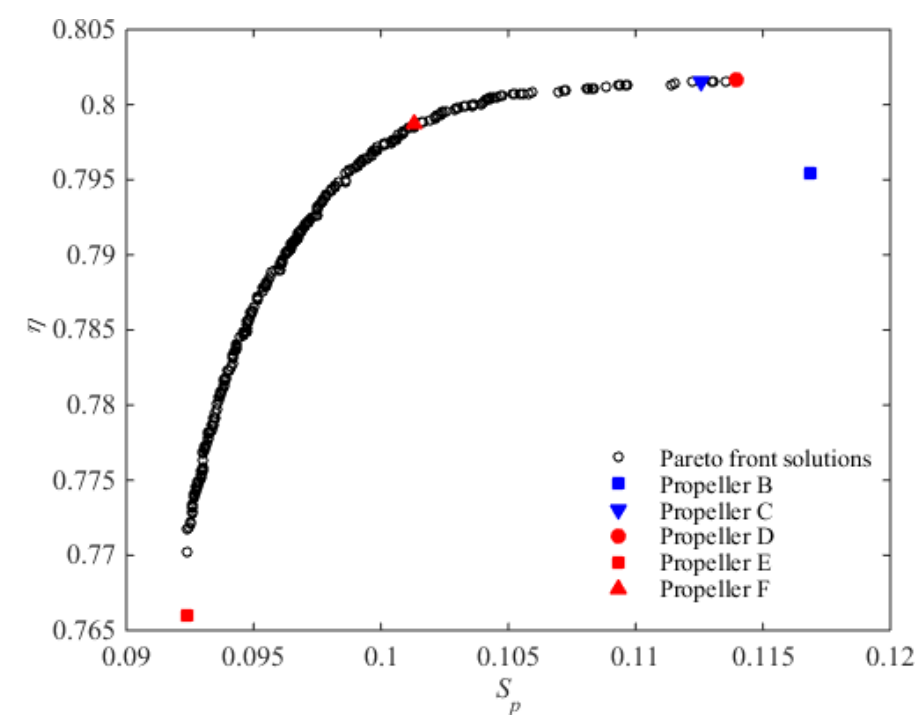

Fig. 27. Pareto frontier of the efficiency and blade area. 
Table 6. Comparison with optimization results of MIGA and NSGA-II.

\begin{tabular}{crccccc}
\hline Propeller & $T(\mathrm{~N})$ & $M /(\mathrm{Nm})$ & $\eta$ & $S_{p}$ & $\Delta \eta$ & \multicolumn{1}{c}{$\Delta S_{p}$} \\
\hline C & 99.93 & 37.21 & $80.15 \%$ & 0.1125 & 0 & \multicolumn{1}{c}{0} \\
$\mathrm{D}$ & 100.04 & 37.25 & $80.17 \%$ & 0.1139 & $+0.02 \%$ & $+1.24 \%$ \\
$\mathrm{E}$ & 101.58 & 39.66 & $76.43 \%$ & 0.0924 & $-3.72 \%$ & $-17.87 \%$ \\
$\mathrm{~F}$ & 99.96 & 37.345 & $79.88 \%$ & 0.1013 & $-0.27 \%$ & $-9.96 \%$ \\
\hline
\end{tabular}

Under the cruise condition of $n=960 \mathrm{rpm}, v_{\infty}=30 \mathrm{~m} / \mathrm{s}$, Propeller D has the same efficiency with Propeller C but the blade area increases by $1.24 \%$. Although the blade area of Propeller E decreases by $17.87 \%$, the efficiency reduces to $76.43 \%$. And Fig. 26] shows that the efficiency of Propeller E obviously decreases under $J<0.9$ and it provides the lower thrust and absorb less energy when the rotational speed of the propeller is fast. Propeller F has the high efficiency and the blade area decreases by $9.96 \%$. From Fig. [26, the aerodynamic performance of Propeller F is close to Propeller B and is superior to Propellers C and D.

Table 7. Comparison with aerodynamic data of Propellers A, B, C, and F.

\begin{tabular}{lrccccc}
\hline Propeller & $T(\mathrm{~N})$ & $M /(\mathrm{Nm})$ & $\eta$ & $S_{p}$ & $\Delta \eta$ & $\Delta S_{p}$ \\
\hline Liu and He [2017] & 98.19 & 39.75 & $73.87 \%$ & 0.1588 & 0 & 0 \\
D'Angelo & 103.45 & 38.81 & $79.54 \%$ & 0.1168 & $+5.67 \%$ & $-26.45 \%$ \\
MIGA & 99.93 & 37.21 & $80.15 \%$ & 0.1125 & $+6.28 \%$ & $-29.16 \%$ \\
NSGA-II & 99.96 & 37.345 & $79.88 \%$ & 0.1013 & $+6.01 \%$ & $-36.21 \%$ \\
\hline
\end{tabular}
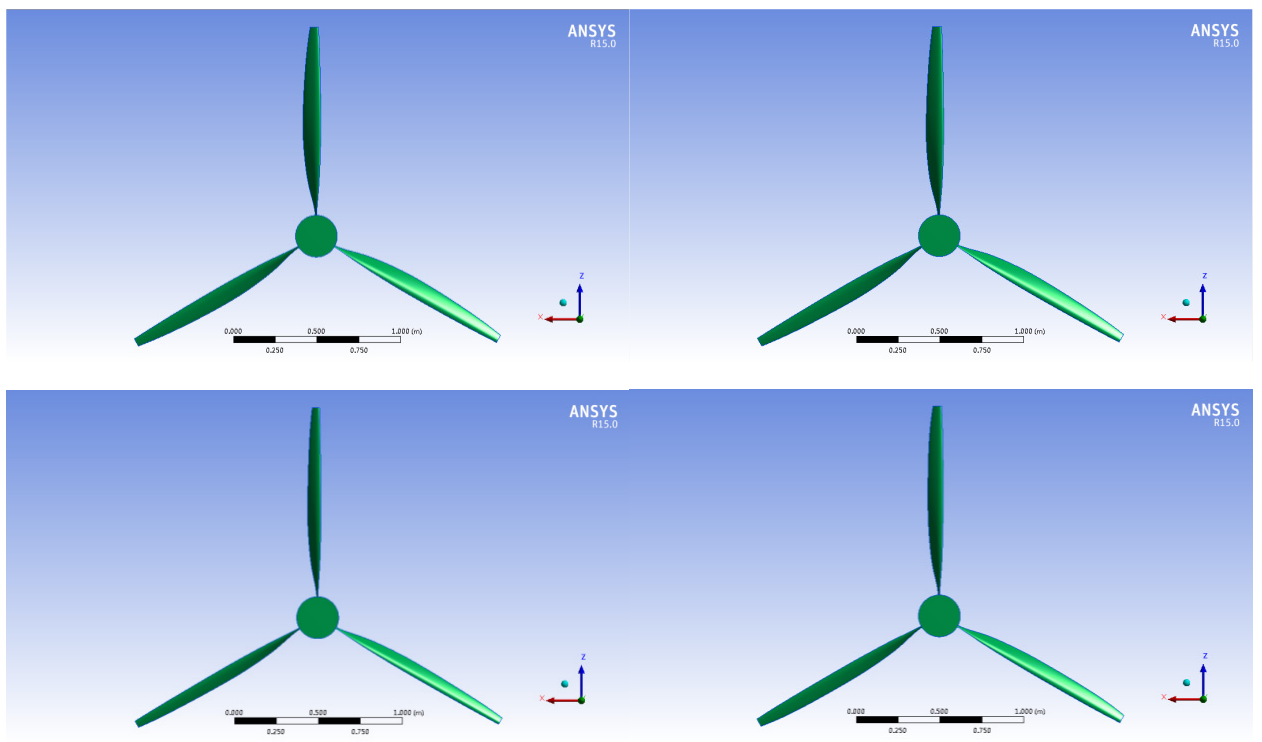

Fig. 28. Solid models of Propellers C, D, E and F. 
The aerodynamic data of the propeller in Liu and $\mathrm{He}$ [2017, the propeller designed by D'Angelo program, and the propellers optimized by improved D'Angelo optimization framework are shown in Table 7 The more reasonable chord and twist angle of the high altitude propeller, compared with Liu and He [2017], are obtained by D'Angelo program. The efficiency increases by $5.67 \%$ for D'Angelo method and the weight of the propeller decreases by $26.45 \%$. The distributions of the chord and twist angle are optimized by MIGA to obtain the highest efficiency propeller. But the efficiency only increases by $0.61 \%$ compared with D'Angelo program. Because the efficiency of D'Angelo program is very high and the distributions of the chord and twist angle are reasonable, so NSGA-II is adopted to seek the balance between the efficiency and the weight. After NSGA-II optimization, the efficiency increases by $6.01 \%$ and the weight decreases by $36.21 \%$.

The solid models of MIGA and NSGA-II optimization are established by CATIA. The three-dimensional models of Propellers C, D, E and F are shown in Fig. 28.

CFD simulation method in Sec. 4 is used to calculate the aerodynamic parameters of Propellers C and F. The performance of Propellers B, C, and D is presented

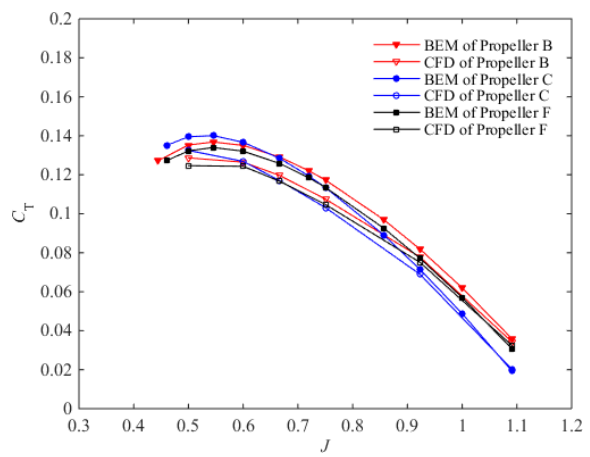

(a)

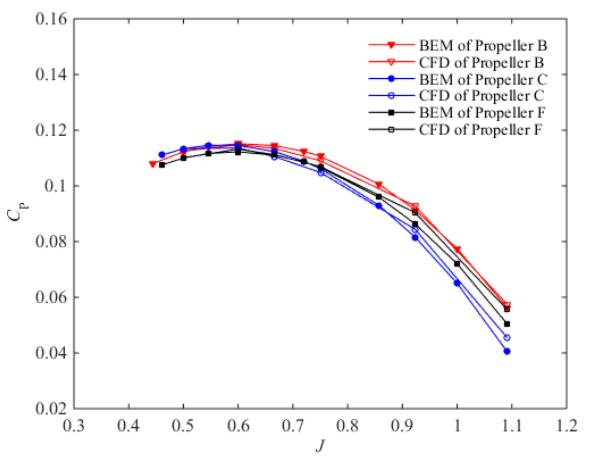

(b)

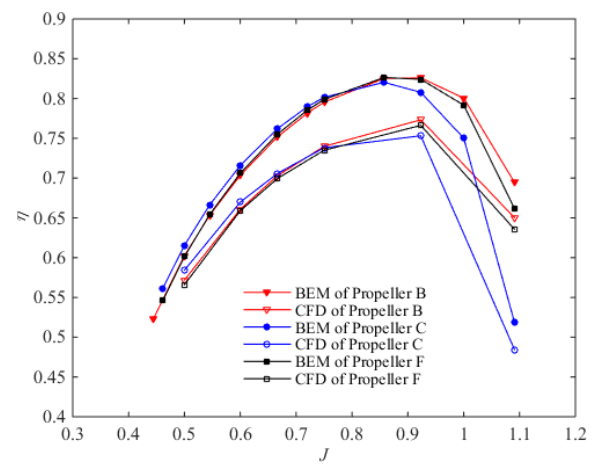

(c)

Fig. 29. Comparison between data calculated by BEM and CFD for Propellers B, C, F: (a) thrust coefficient, (b) power coefficient, (c) propeller efficiency. 
in Fig. 29, It is shown that the results of BEM are in fair agreement with CFD simulations. BEM predicts a slightly larger thrust coefficient than CFD and the power coefficient of BEM is close to the CFD results. The results also show that when the advance ratio $J=0.75$, the maximum efficiency difference between BEM calculation and CFD simulation for Propeller B is $5.55 \%$. When the advance ratio $J=0.75$, the maximum efficiency difference between BEM calculation and CFD simulation for Propeller $\mathrm{C}$ is $6.34 \%$. When the advance ratio $J=0.75$, the maximum efficiency difference between BEM calculation and CFD simulation for Propeller F is $6.39 \%$.

Under the cruise condition of $n=960 \mathrm{rpm} v_{\infty}=30 \mathrm{~m} / \mathrm{s}$, the pressure distributions on the blade surfaces of Propellers B, C, and F are shown in Figs. 30 31

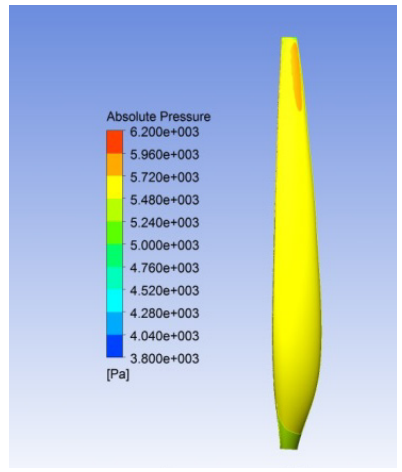

(a)

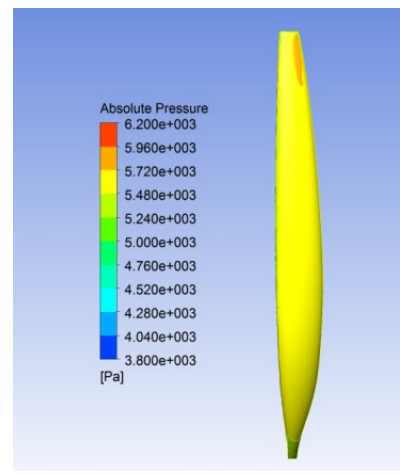

(b)

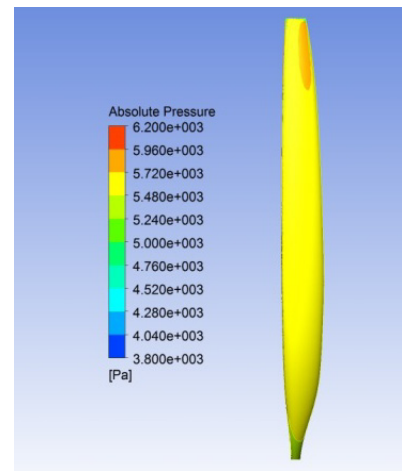

(c)

Fig. 30. Comparison of pressure distribution on lower surfaces for $n=960 \mathrm{rpm}$ and $v_{\infty}=30 \mathrm{~m} / \mathrm{s}$. (a) Propeller B, (b) Propeller C, and (c) Propeller F.

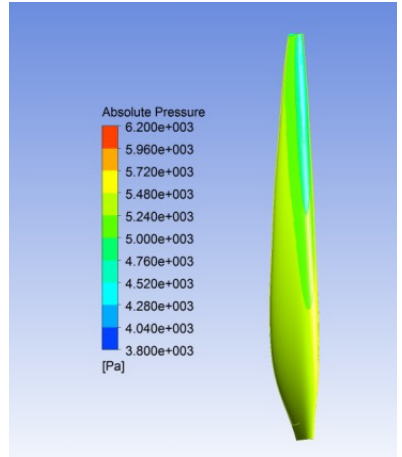

(a)

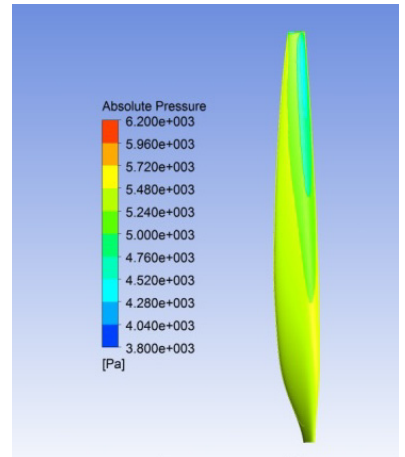

(b)

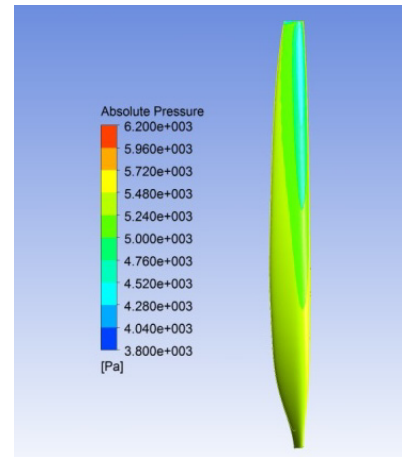

(c)

Fig. 31. Comparison of pressure distribution on upper surfaces for $n=960 \mathrm{rpm}$ and $v_{\infty}=30 \mathrm{~m} / \mathrm{s}$. (a) Propeller B, (b) Propeller C, and (c) Propeller F. 
When the propeller is rotating, the pressure of the upper surface is lower and the lower surface is higher. Thus, the pressure difference between the upper surface and lower surface can produce the thrust. The pressure of the lower surface gradually increases from the root to the tip of Propeller B and the maximum pressure is between $0.8 R$ and $0.96 R$. The maximum pressure of Propeller $\mathrm{C}$ is between $0.85 R$ and $0.96 R$ and Propeller $\mathrm{F}$ is between $0.82 R$ and $0.96 R$. It is indicated that the thrust of Propeller B is larger than Propeller F and the thrust of Propeller C is lower than Propeller F.

\section{Conclusion}

This paper proposes that the improved D'Angelo optimization framework is used to design and optimize a high altitude propeller. This framework adopts multilevel optimization strategy. The first-level optimization can obtain the high efficiency D'Angelo propeller whose aerodynamic performance is verified by CFD simulation and BEM theory. The second-level optimization uses the surrogate model and optimization algorithm to obtain the high efficiency and light weight propeller. In the second-level optimization MIGA method is applied to obtain the maximum efficiency propeller by changing the chord and twist angle distributions of the D'Angelo propeller. Then NSGA-II method is introduced and used to solve the multi-objective optimization problem acquiring the high efficient and lightweight propeller. The Pareto frontier solutions between the efficiency and the blade area are acquired and discussed in detail. Besides, the aerodynamic parameters of these three propellers are calculated by BEM theory compared with the CFD simulations. We can draw some conclusions on the high altitude propeller:

(1) The improved D'Angelo optimization framework can be applied to design the high altitude propeller and we can obtain the high efficient and lightweight propeller.

(2) In the second-level optimization MIGA method can improve the D'Angelo propeller efficiency, but the efficiency is not obviously enhanced. Because the D'Angelo method has taken full advantage of the aerodynamic efficiency of the section airfoil, so it is very difficult to improve the propeller efficiency by changing the chord and twist angle distributions. However, the reasonable chord and twist angle distributions are obtained.

(3) The blade area of the propeller can be decreased significantly at the expense of reducing a little efficiency by NSGA-II. NSGA-II method can obtain the high efficiency and lighter propeller.

(4) The aerodynamic characteristics calculated by CFD simulations are in fair agreement with the BEM results. It is indicated that the improved D'Angelo optimization framework is feasible and the multilevel optimization strategy is effective and valid for the high altitude propeller design. Besides, the optimal propeller can reach the cruise requirements of the stratospheric aircraft at the design point. 


\section{References}

Adkins, C. N. and Liebeck, R. H. [1994] "Design of optimum propellers," J. Propul. Power 10(5), 676-682.

Betz, A. and Prandtl, L. [1919] "Schraubenpropeller mit geringstem energieverlust," Göttinger Nachrichten 1919, 193-217.

Berci, M., Toropov, V. V., Hewson, R. W. and Gaskell, P. H. [2014] "Multidisciplinary multifidelity optimisation of a flexible wing aerofoil with reference to a small UAV," Struct. Multidiscipl. Opt. 50(4), 683-699.

Bekele, E. G. and Nicklow, J. W. [2007] "Multi-objective automatic calibration of SWAT using NSGA-II," J. Hydrol. 341(3), 165-176.

Colozza, A. [2003] "Initial feasibility assessment of a high altitude long endurance airship," NASA/CR-2003-212724, 12.

D'Angelo, S., Berardi, F. and Minisci, E. [2002] "Aerodynamic performances of propellers with parametric considerations on the optimal design," Aeronaut. J. 106(1060), 313320.

Dorfling, J. and Rokhsaz, K. [2014] "Constrained and unconstrained propeller blade optimization," J. Aircraft 52(4), 1179-1188.

Drela, M. [1989] "XFOIL: An analysis and design system for low reynolds number airfoils," in Low Reynolds Number Aerodynamics, ed. Mueller, T. J., Lecture Notes in Engineering, Vol. 54 (Springer, Berlin, Heidelberg), pp. 1-12.

Goldstein, S. [1929] "On the vortex theory of screw propellers," Proc. R. Soc. Lond. 123(792), 440-465.

Glauert, H. [1963] Airplane Propellers. Aerodynamic Theory (Dover, New York), pp. 251268.

Hou, S., Zheng, Y., Xie, J. and Han, X. [2014] "Optimization design of NJ shaped guardrail based on collision safety consideration," Int. J. Comput. Methods 11(6) 1350083.

Jiao, J. et al. [2018] "Optimal design and experiment of propellers for high altitude airship," J. Aerospace Eng. 232(10), 1887-1902.

Jin, R., Chen, W. and Simpson, T. W. [2001] "Comparative studies of metamodelling techniques under multiple modelling criteria," Struct. Multidiscipl. Optim. 23(1), 1-13.

Kwon, H. I. et al. [2015] "Design of efficient propellers using variable-fidelity aerodynamic analysis and multilevel optimization," Journal of Propulsion and Power 31(4), 10571072.

Liu, X. Q. and He, W. L. [2017] "Performance calculation and design of stratospheric propeller," IEEE Access 5, 14358-14368.

Larrabee, E. E. [1979] "Practical design of minimum induced loss propellers," Soc. Automotive Engineers TP-790585, Warrendale, PA.

Li, B., Lu, H. and Deng, S. H. [2015] "Validation of an actuator disk model for numerical simulation of propeller," J. Aerospace Eng. 229(8), 1454-1463.

Meng, J. H., Hu, J., Xiao, H. D. and Lv, M. Y. [2017] "Hierarchical optimization of the composite blade of a stratospheric airship propeller based on genetic algorithm," Struct. Multidiscipl. Optim. 56(6), 1341-1352.

Morgado, J. et al. [2015] "High altitude propeller design and analysis," Aerospace Science and Technology 45, 398-407.

McCrink, M. H. and Gregory, J. W. [2017] "Blade element momentum modeling of lowreynolds electric propulsion systems," J. Aircraft 54(1), 163-176.

Morgado, J., Silvestre, M. Â. R. and Páscoa, J. C. [2014] "Validation of new formulations for propeller analysis," Journal of Propulsion and Power 31(1), 467-477.

Mieloszyk, J., Galiński, C. and Piechna, J. [2013] "Contra-rotating propeller for fixed wing MAV: part 1," Aircraft Engineering and Aerospace Technology 85(4), 304-315. 
Mackay, M. D., Beckman, R. J. and Conover, W. J. [1979] "A comparison of three methods for selecting values of input variables in the analysis of output from a computer code," Technometrics 21, 239-245.

Ma, R. and Liu, P. Q. [2009] "Numerical simulation of low-Reynolds-number and high-lift airfoil S1223," Proc. World Congress on Engineering, London, UK.

Noll, T. E. et al. [2004] "Investigation of the Helios prototype aircraft mishap volume I mishap report," Helios Mishap Investigation Board, NASA Langley Research Center, Hampton, VA, Jan. 2004, pp. 1-100.

Qin, D., Pan, G., Huang, Q., Zhang, Z. and Ke, J. [2018] "Numerical investigation of different tip clearances effect on the hydrodynamic performance of pumpjet propulsor," Int. J. Comput. Methods 15(5), 1850037.

Rahmati, M. T. [2009] "Application of a pressure correction method for modeling incompressible flow through turbomachines," Int. J. Comput. Methods 6(3), 399-411.

Romeo, G. et al. [2012] "Design and testing of a propeller for a two-seater aircraft powered by fuel cells," J. Aerospace Eng. 226(7), 804-816.

Romeo, G., Moraglio, I. and Novarese, C. [2007] "ENFICA-FC: Preliminary survey \& design of 2-seat aircraft powered by fuel cells electric propulsion," 7th AIAA Aviation Technology, Integration and Operations Conf., Belfast, Northern Ireland, Vol. 1, Paper AIAA-2007-7754, pp. 602-616.

Sodja, J., Stadler, D. and Kosel, T. [2012] "Computational fluid dynamics analysis of an optimized load-distribution propeller," J. Aircraft 49(3), 955-961.

Selig, M. S. and Guglielmo, J. J. [1997] "High-lift low Reynolds number airfoil design," J. Aircraft 34(1), 72-79.

Tang, Z. H., Liu, P. Q., Chen, Y. X. and Guo, H. [2015] "Experimental study of counterrotating propellers for high-altitude airships," Journal of Propulsion and Power 31(5), 1491-1496.

Yin, H., Wen, G. and Gan, N. [2011] "Crashworthiness design for honeycomb structures under axial dynamic loading," Int. J. Comput. Methods 8(4) 863-877.

Zhang, M. C. et al. [2017] "Multidisciplinary design and multi-objective optimization on guide fins of twin-web disk using Kriging surrogate model," Struct. Multidiscipl. Optim. 55(1), 361-373.

Zhao, D. J. et al. [2015] "Optimization of suction control on an airfoil using multi-island genetic algorithm," Procedia Eng. 99, 696-702.

Zheng, X. K. et al. [2017] "The efficiency analysis of high-altitude propeller based on vortex lattice lifting line theory," Aeronautical Journal 121(1236), 141-162.

Zhang, T. T., Huang, W., Wang, Z. G. and Yan, L. [2016] "A study of airfoil parameterization, modeling, and optimization based on the computational fluid dynamics method," Journal of Zhejiang University-SCIENCE A 17(8), 632-645. 